\title{
Enhanced Energy Detection using Matched Filter for Spectrum Sensing in Cognitive Radio Networks
}

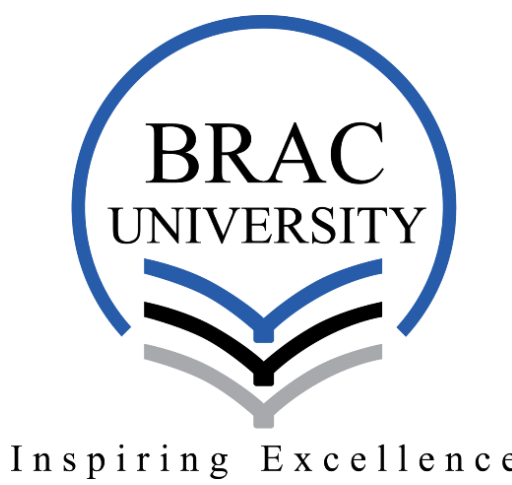

SUBMISSION DATE: 16.04.2018

SUBMITTED BY:

\author{
Umme Salama (14101041) \\ Pramit Likhan Sarker (13201073) \\ Department of Computer Science and Engineering
}

\section{Supervisor:}

Amitabha Chakrabarty, Ph.D

Assistant Professor

Department of Computer Science and Engineering 


\section{Declaration}

We, hereby declare that this thesis is based on results we have found ourselves. Materials of work from researchers conducted by others are mentioned in references.

Amitabha Chakrabarty, Ph.D

Assistant Professor

Umme Salama (14101041)

Department of Computer Science

and Engineering

BRAC University 


\begin{abstract}
Cognitive Radio (CR) deals with the designing of intelligent wireless communication systems through the use of transceivers that are capable of automatically detecting and accessing vacant communication channels in the radio bandwidth while avoiding the ones occupied, with the aim of maximizing the utilization of the Radio Frequency (RF) spectrum and minimizing the interference of users. In primary transmitter detection i.e. noncooperative spectrum sensing, the licensed primary users (PUs) are detected based on the signal received by the unlicensed secondary users (SUs). This paper provides an insight into one such method, namely, the energy detection technique, which has low computational and implementation complexities, and is extremely generic. However, the detection of weak PU signals across a noisy channel is a challenging endeavor and calls for a more sophisticated approach. A matched filter can be used to obtain additional information regarding the channel activity, help individuate the transmitted pulses from the noise and reduce the effects of unlicensed signal interference. The proposed algorithm attains results from a matched filter and implements it within the energy detector, analyzing the signals over an Additive White Gaussian Noise (AWGN) channel for a range of Signal-to-Noise Ratios (SNRs), which are then evaluated through Receiver Operating Characteristic (ROC) curves with probability of detection $\left(\mathrm{P}_{\mathrm{d}}\right)$ and probability of false alarm $\left(\mathrm{P}_{\mathrm{f}}\right)$ as performance metrics.
\end{abstract}

Keywords: spectrum sensing, matched filter, energy detection, primary user signal, signal-to-noise ratio, energy threshold 


\section{Acknowledgement}

First and foremost we wish to thank the Almighty for bestowing upon us the ability, opportunity and the necessary resources to complete our undergraduate dissertation, and thereby blessing us with a chance to attain a great deal of knowledge. This report is above all, the result of His kindness towards us, and to Him we would like to express our deepest gratitude.

We are and will remain irrevocably indebted towards our thesis supervisor, Dr. Amitabha Chakrabarty, Assistant Professor of the Department of Computer Science and Engineering, BRAC University, for encouraging us in all our scientific endeavors, providing us with his most valuable consultations and critiques on all the parts of our dissertation, during all the stages of its preparation. It was an honor, a privilege and an absolute pleasure to be working under his supervision, for one could simply not wish for a mentor more proficient, kinder or more spirited than him. We thank him for planting the idea of our thesis topic in our minds, and for generously supporting us through the tumultuous ups and downs of the semesters past, till the very end of this unforgettable journey.

We are immensely thankful for the opportunity to consult Dr. Nafees Mansoor, Assistant Professor of the Department of Computer Science and Engineering, University of Liberal Arts Bangladesh, whose expertise and insights allowed us to learn a great deal more. We will always be obliged for the kindness and generosity with which he treated us, and the enlightening conversations that we were fortunate enough to have with him.

We will forever be grateful to Dr. Saifur Rahman Sabuj, Assistant Professor of the Department of Electrical and Electronic Engineering, BRAC 
University, for the profound guidance, unfailing support, and the relentless dedication with which he guided us at our times of distress. His invaluable advice has enabled the successful completion of our work, and we would like to express our heartfelt gratitude towards him for granting us time for discussion and for patiently seeing us through the trials that we faced.

Finally, we would like to convey our unbounded respect and love towards our parents for supporting us unconditionally throughout our years of study, and through the process of researching and writing this paper. We choose this moment to acknowledge our indebtedness and deep sense of gratitude towards them. To them we owe our lives, and to them we dedicate this dissertation. 


\section{Table of Contents}

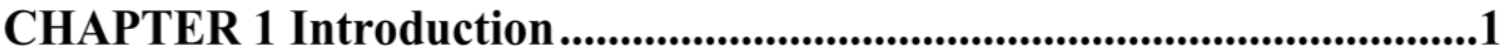

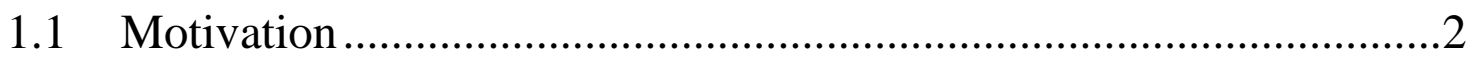

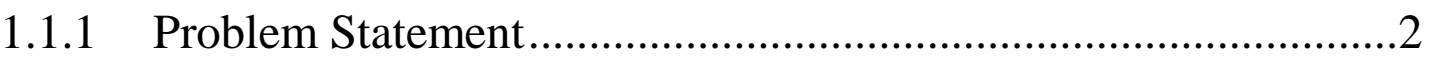

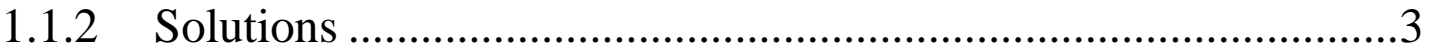

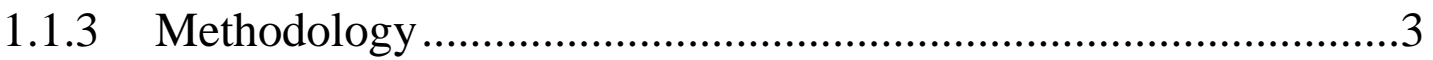

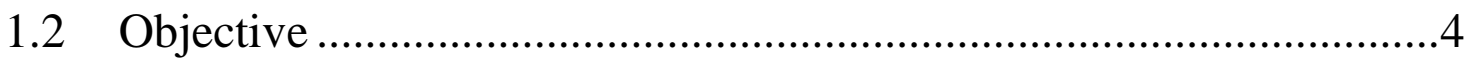

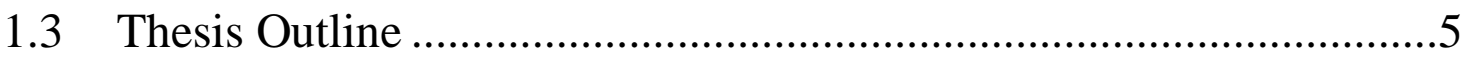

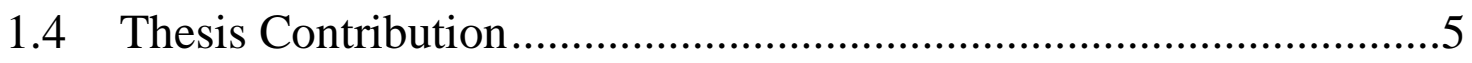

CHAPTER 2 Literature Review .............................................................6

2.1 Wireless Communication Systems …........................................6

2.1.1 Licensed and Unlicensed Spectrum.......................................

2.1.2 Software Defined Radio .........................................................

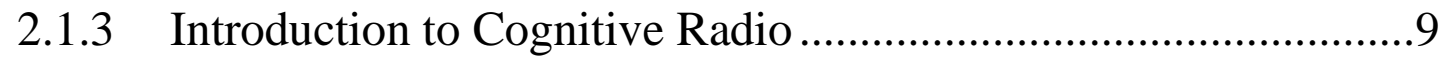

2.1.4 Primary and Secondary Users.................................................10

2.2 Cognitive Radio Features and Potential..........................................11

2.2.1 Cognitive Radio Architecture …...............................................12

2.2.2 Cognitive Radio Interfaces ...................................................14

2.2.3 Dynamic Spectrum Access …..................................................14

2.2.4 Functions of Cognitive Radio .................................................15

2.2.5 Cognitive Radio Components ..................................................16 
2.3 Spectrum Sensing in Cognitive Radio

2.3.1 Non-Cooperative Spectrum Sensing ......................................19

2.3.2 Cooperative Spectrum Sensing ................................................20

2.3.3 Interference-Based Spectrum Sensing .....................................20

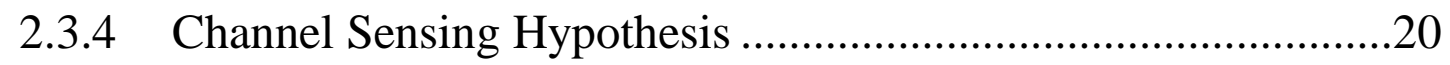

CHAPTER 3 Enhanced Energy Detection Using Matched Filter ...........22

3.1 Conventional Energy Detection Algorithm ....................................22

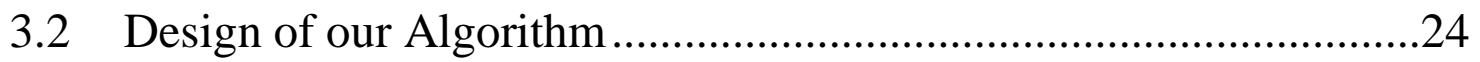

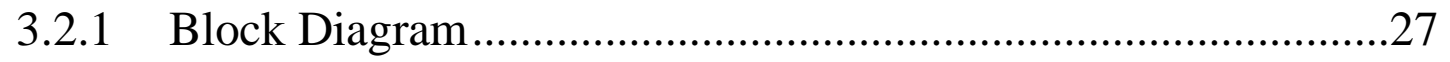

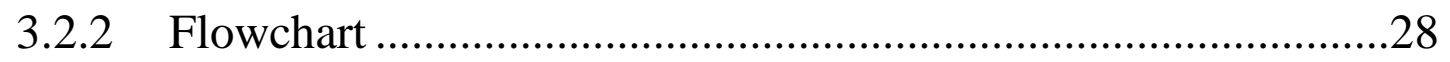

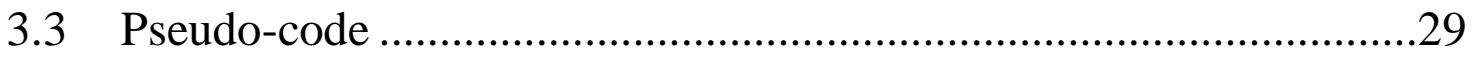

3.4 Simulation Setup using (CMATLAB 2017a ..................................29

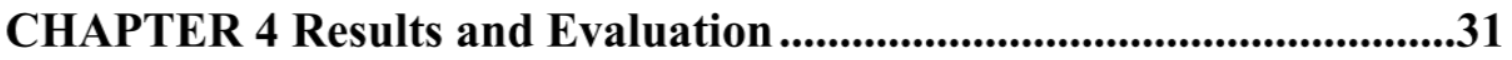

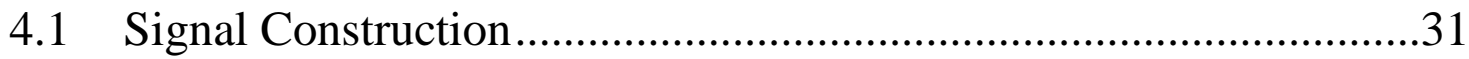

4.2 Cross-correlation of Signal and Matched Filter Template ...............31

4.3 Signal Energy and Energy Threshold ..............................................33

$4.4 \mathrm{P}_{\mathrm{d}}$ vs. $\mathrm{P}_{\mathrm{f}}$ of the Algorithms at a Fixed SNR Value............................34

4.5 $\mathrm{P}_{\mathrm{m}}$ vs. $\mathrm{P}_{\mathrm{f}}$ of the Algorithms at a Fixed SNR Value ...........................35

4.6 ROC Curves for a Range of SNR Values .......................................36

CHAPTER 5 Conclusion and Future Work .............................................42

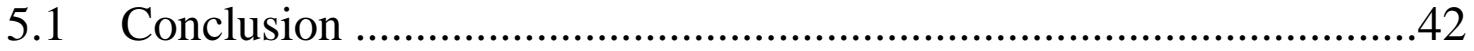


5.2 Future Work.

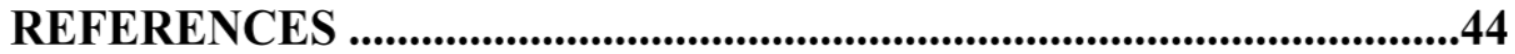




\section{List of Figures}

Figure 1: Radio Spectrum allocated for Wireless Communications ..............6

Figure 2: Cognitive Radio Network architecture ........................................10

Figure 3: Cognitive Radio protocol stack...................................................13

Figure 4: External interfaces of Cognitive Radio..........................................14

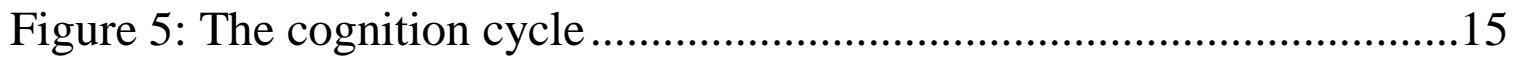

Figure 6: Components in a Cognitive Radio System ..................................16

Figure 7: Spectrum hole (or spectrum opportunity) ...................................17

Figure 8: Types of Spectrum Sensing .....................................................18

Figure 9: Block diagram for Enhanced Energy Detection Algorithm using

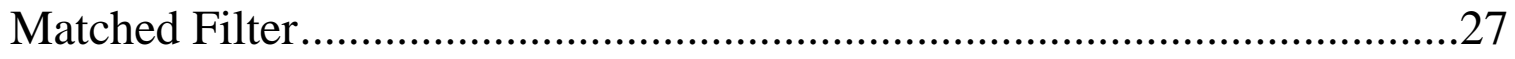

Figure 10: Flowchart for Enhanced Energy Detection Algorithm using

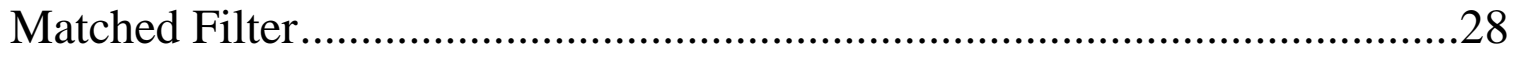

Figure 11: Construction of the Received Signal............................................31

Figure 12: Received Signal before and after Cross-correlation ....................32

Figure 13: Energy Comparison of the Algorithms with Threshold ..............33

Figure 14: $\mathrm{P}_{\mathrm{d}}$ vs. $\mathrm{P}_{\mathrm{f}}$ for the Algorithms at a Fixed SNR ................................34

Figure 15: $\mathrm{P}_{\mathrm{m}}$ vs. $\mathrm{P}_{\mathrm{f}}$ for the Algorithms at a Fixed SNR ................................35

Figure 16: ROC Curves for Conventional Energy Detection at Different SNRs

Figure 17: ROC Curves for Enhanced Energy Detection using Matched Filter at Different SNRs .37 


\section{List of Tables}

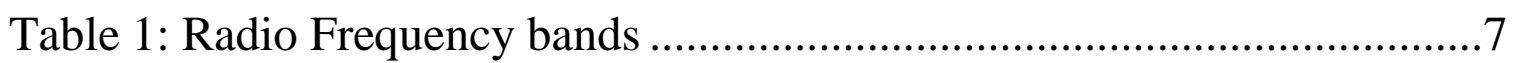

Table 2: Summary of the Non-Cooperative Spectrum Sensing techniques ..19

Table 3: Channel Sensing Hypothesis in Spectrum Sensing........................21 


\section{CHAPTER 1}

\section{Introduction}

Spectrum scarcity is fast becoming a critical global issue in the field of wireless communication due to the increasingly high demand for the use of the limited natural resource that is the electromagnetic RF spectrum. Static allocation of the spectrum bands leads to the underutilization of the available spectrum, and since wireless operators are allotted exclusive licenses for operating in particular frequency bands, the conventional approach to spectrum management is notoriously stringent. CR technology comes as a solution which recompenses greatly for the problem of spectral congestion and scarcity through opportunistic usage of the RF bands that are left vacant by the licensed PUs, and through enabling Dynamic Spectrum Access (DSA) which leads to an efficient use of the scarce resource in question.

One of the most imperative aspects of the CR technology according to [1] is the ability to measure, sense, learn, and be aware of the parameters related to the radio channel characteristics, including knowledge about the availability of spectrum and power, networks and nodes, the entire infrastructure as so we speak; the user requirements, local policies and the operating precincts. Spectrum sensing can thus be defined as the task of obtaining cognizance regarding the spectrum occupancy, as well as perceiving the presence and activity or transmissions of the PUs within a particular licensed spectrum at one location, which can be obtained by using geolocation and database, beacons, or by local spectrum sensing at CRs. 


\subsection{Motivation}

Energy detection is the most conventionally used spectrum sensing technique, predominantly for each potential signal to be detected without any prior knowledge, despite its menial performance compared to that of other non-cooperative sensing techniques like matched filter detection and cyclostationary feature detection. Since an energy detector is unable to differentiate the target signal from intrusive signals, the SUs must be silenced while it operates, and when loss of control messages and time synchronization errors occur, the coordination becomes subject to fiascos. Some SUs may then transmit even during a silent period, causing a false alarm at the energy detector if no PU signals are present [2]. Keeping this perception in mind, with the hopes of formulating a plausible solution to the problem of unlicensed interference in transmitter detection, we have chosen this topic in particular for our dissertation.

\subsubsection{Problem Statement}

The purpose of spectrum sensing is to determine the status of the spectrum and the transmission of signals of the PUs by periodically sensing the target frequency band and the incumbent operations [3]. However, the energy detector is vulnerable to the ambiguity of noise power or variance, and it can merely detect the presence of a signal but cannot differentiate its type. For instance, if signals from SUs share the same channel with the PU, without having any prior knowledge about the signal that is transmitted, a CR may well be unaware of its source and be unable to make this distinction. So the detection error would be high in the presence of signal sources that are not the 
licensed PUs, detecting the transmitted pulses in the noisy received signal would turn out to be highly problematic. All in all, the energy detection technique may not always be insured and its performance may even prove to be inadequate in comparison to other non-cooperative spectrum sensing techniques by nature, therefore it requires the appendage of others.

\subsubsection{Solutions}

When the information of the signal from a licensed user is known, the modulation and packet format, a matched filter is the optimal linear filter for maximizing the SNR in the presence of additive stochastic noise, which here in the case of our dissertation, is stationary Gaussian noise. Hence, our proposed algorithm cartels the result from a matched filter and implements it within the energy detector for a low SNR regime. It strives to upgrade the performance of energy detection after comparing a known signal, i.e. the template of the PU signal, with the received signal of the unlicensed SU through cross-correlation. Our algorithm requires minimal changes in hardware and therefore can be implemented; in fact, the international standard IEEE 802.11 already uses matched filtering to detect incoming packets, which aids in the maintenance of packet synchronization [2] - these built-in matched filters can be used to improve the performance of spectrum sensing in CRs.

\subsubsection{Methodology}

We constructed the matched filter template by making use of the Phased Array System Toolbox in CMATLAB 2017a, cross-correlation between the template and the received signal by the SU was done to create the final signal 
and energy which was to be the test statistic. Keeping in mind that the test statistic was obtained from a fixed number of samples and an SNR value, we considered the conventional method of energy detection with an energy threshold under Constant False Alarm Rate (CFAR) in [4] which we compared the test statistic with. After calculating the $\mathrm{P}_{\mathrm{d}}$, we generated the ROC curve, which is a graphical plot illustrating the investigative ability of a binary classifier system (PU present or PU absent) while its distinguishing

threshold is varied. ROC curves for a range of low SNRs were also produced, and a number of comparisons drawn between the conventional energy detection algorithm and the enhanced algorithm aided by matched filter. The developed algorithm had been tested extensively before the writing of this paper commenced.

\subsection{Objective}

As for the parameters allied with spectrum sensing, it can be said that the higher the $\mathrm{P}_{\mathrm{d}}$, the better the PUs are protected from unlicensed interference. Through the SUs' perspective, the lower the $\mathrm{P}_{\mathrm{f}}$, the more the chances of the channel being reused while it is available [5]. Our objective therefore is to explore the Energy detection technique, and leverage a matched filter prior to the energy threshold comparison in a noisy environment, in order to enhance the performance and reduce unlicensed interference. Our goal is to obtain the best ROC curve possible with the maximum $\mathrm{P}_{\mathrm{d}}$ and minimum $\mathrm{P}_{\mathrm{f}}$. 


\subsection{Thesis Outline}

Various aspects of spectrum sensing shall be discussed throughout this thesis dissertation, and the paper will comprise of five different chapters, the outlines of which are given as follows. Starting with the introduction of spectrum sensing in CR networks in Chapter 1, the challenges of unsolicited interference between the PUs and SUs are discussed. We propose a novel approach to solving them and outline what we aspire to achieve with our algorithm. The spectrum sensing concepts and a review of all the background research done in pursuance of devising our algorithm is defined in Chapter 2. The enhanced algorithm is proposed and its design explained in Chapter 3, along with its overall system implementation. Chapter 4 explains the analysis of the results obtained and the evaluations made. Finally, the concluding remarks related to our work are explained and the plans and scope for future works are presented in Chapter 5.

\subsection{Thesis Contribution}

The first part of the thesis consists of a literature review of important background knowledge, an in-depth survey of which has been carried out for comparative study. We have formulated the novel algorithm in order to overcome the limitations of the energy detection technique and utilize the advantages of the matched filter, although it is heavily dependent upon the energy threshold formula by Dr. Ying-Chang Liang in [5]. The code is based on the conventional energy detector, the modifications that have been made were implemented through the computing environment of CMATLAB. 


\section{CHAPTER 2}

\section{Literature Review}

This chapter contains the necessary information gathered from all of the background research that has been conducted, and the related existing work summaries.

\subsection{Wireless Communication Systems}

The basic building blocks of radio communication are radio waves, and waves with different frequencies have different propagation characteristics, each of which is suitable for a specific wireless application. The range of 3 $\mathrm{kHz}$ to $300 \mathrm{GHz}$ is primarily used for wireless communication worldwide, and assigned to different licensed entities by the government.

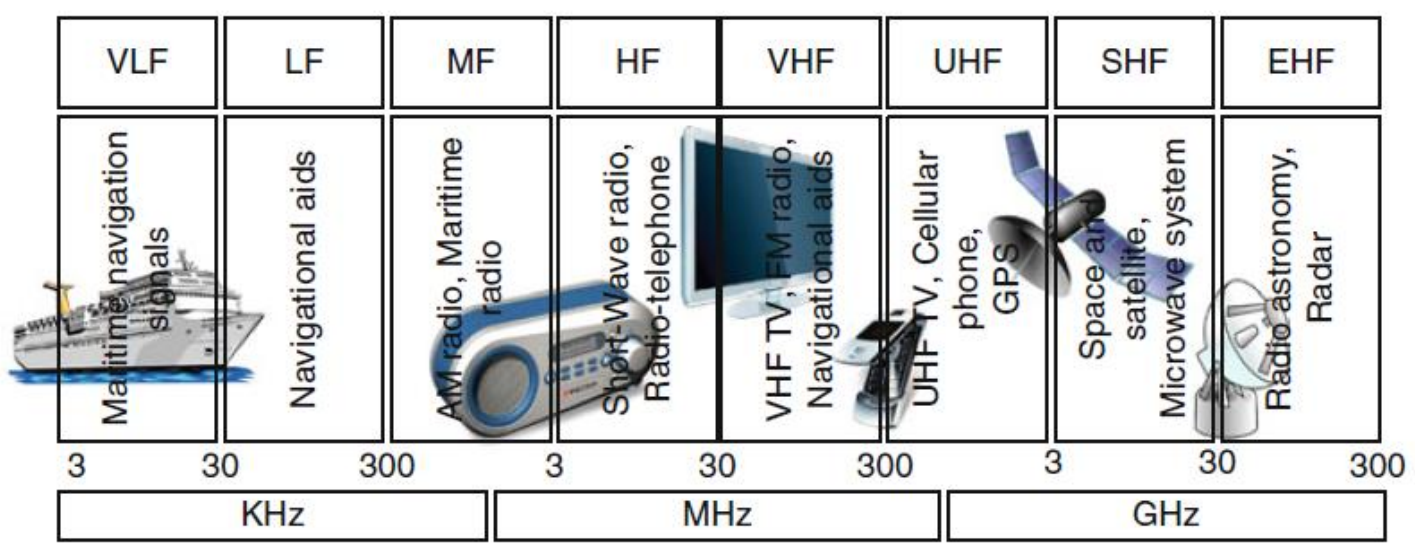

Figure 1: Radio Spectrum allocated for Wireless Communications [6]

The various wireless communications systems available today differ in terms of data rate of transmission, geographical coverage area, transmission power, and mobility support for users. However, the challenges including radio resource allocation/management and medium access control, rate 
control, handoff and mobility management, quality of service (QoS) provisioning, and security unanimously exist for all [3].

Table 1: Radio Frequency bands [6]

\begin{tabular}{|l|l|l|l|}
\hline Abbreviation & Designation & $\begin{array}{l}\text { Frequency } \\
\text { Range }\end{array}$ & $\begin{array}{l}\text { Uses in Wireless } \\
\text { Communications }\end{array}$ \\
\hline ELF & $\begin{array}{l}\text { Extremely low } \\
\text { frequency }\end{array}$ & $3 \mathrm{~Hz}-30 \mathrm{~Hz}$ & - \\
\hline SLF & $\begin{array}{l}\text { Super low } \\
\text { frequency }\end{array}$ & $30 \mathrm{~Hz}-300 \mathrm{~Hz}$ & - \\
\hline ULF & $\begin{array}{l}\text { Ultralow } \\
\text { frequency }\end{array}$ & $\begin{array}{l}300 \mathrm{~Hz}-3000 \\
\mathrm{~Hz}\end{array}$ & - \\
\hline VLF & $\begin{array}{l}\text { Very low } \\
\text { frequency }\end{array}$ & $3 \mathrm{kHz}-30 \mathrm{kHz}$ & $\begin{array}{l}\text { Maritime navigation } \\
\text { signals }\end{array}$ \\
\hline LF & Low frequency & $\begin{array}{l}30 \mathrm{kHz}-300 \\
\mathrm{kHz}\end{array}$ & Navigational aids \\
\hline MF & $\begin{array}{l}\text { Medium } \\
\text { frequency }\end{array}$ & $\begin{array}{l}300 \mathrm{kHz}-3000 \\
\mathrm{kHz}\end{array}$ & $\begin{array}{l}\text { AM radio, Maritime } \\
\text { radio }\end{array}$ \\
\hline VHF & $\begin{array}{l}\text { High } \\
\text { frequency }\end{array}$ & $\begin{array}{l}3 \mathrm{MHz}- \\
30 \mathrm{MHz}\end{array}$ & $\begin{array}{l}\text { Short-Wave radio, } \\
\text { Radio-telephone }\end{array}$ \\
\hline UHF & $\begin{array}{l}\text { Very high } \\
\text { frequency }\end{array}$ & $\begin{array}{l}30 \mathrm{MHz}- \\
300 \mathrm{MHz}\end{array}$ & $\begin{array}{l}\text { VHF TV, FM radio, } \\
\text { Navigational aids }\end{array}$ \\
\hline SHF & $\begin{array}{l}\text { Ultra high } \\
\text { frequency }\end{array}$ & $\begin{array}{l}300 \mathrm{MHz}- \\
3000 \mathrm{MHz}\end{array}$ & $\begin{array}{l}\text { UHF TV, Cellular } \\
\text { phone, GPS }\end{array}$ \\
\hline EHF & $\begin{array}{l}\text { Super high } \\
\text { frequency }\end{array}$ & $\begin{array}{l}3 \mathrm{GHz}-30 \\
\mathrm{GHz}\end{array}$ & $\begin{array}{l}\text { Space and satellite, } \\
\text { Microwave system }\end{array}$ \\
\hline & $\begin{array}{l}\text { Extremely high } \\
\text { frequency }\end{array}$ & $\begin{array}{l}30 \mathrm{GHz}-300 \\
\mathrm{GHz}\end{array}$ & $\begin{array}{l}\text { Radio astronomy, } \\
\text { Radar }\end{array}$ \\
\hline
\end{tabular}

\subsubsection{Licensed and Unlicensed Spectrum}

The apparent spectrum under-utilization has primarily driven CR technology, which is a new and emerging concept in wireless access. The two types of CR networks are distinguished based on the spectrum bands, namely 
the licensed and the unlicensed bands. The Unlicensed bands include Industrial, Scientific and Medical (ISM) bands such as 902-928MHz, 2.4$2.5 \mathrm{GHz}$, and $5.725-5.875 \mathrm{GHz}$. Furthermore, ISM bands are also shared with non-ISM applications, e.g., Bluetooth: 2.402-2.48GHz; IEEE 802.11/WiFi: 2.45 and $5.8 \mathrm{GHz}$ bands; and IEEE 802.15.4, ZigBee and other personal area networks: $915 \mathrm{MHz}$ and $2.45 \mathrm{GHz}$ bands - these bands can be utilized by CRs. For the licensed bands on the other hand, the spectrum is licensed into different applications, e.g., aeronautical and maritime communications: 300$535 \mathrm{kHz}$; AM radio: $535 \mathrm{kHz}$ and $1.605 \mathrm{MHz}$; and LTE-North America: 700 $\mathrm{MHz}, 800 \mathrm{MHz}, 1.9 \mathrm{GHz}, 1.7 / 2.1 \mathrm{GHz}$ and $2.6 \mathrm{GHz}$ [6]. One instance of the CR technology trying to justifiably overcome the under-utilization of spectrum can be seen from the Wireless Regional Area Network (WRAN) standard which operates in unused television (TV) channels in 698-806MHz.

\subsubsection{Software Defined Radio}

The Federal Communications Commission (FCC) defines a Software Defined Radio as "a device in which the operating parameters are controlled by software, allowing the radio to be programmed to transmit and receive on a variety of frequencies and/or to use one or more different transmission formats supported by its hardware design [7]." Therefore the frequency range, modulation type, maximum output power, as well as the circumstances under which the transmitter operates, in accordance with Commission rules, can be altered for a Software Defined Radio (SDR) by making a change in software without making any changes to hardware components that affect the RF emissions. 


\subsubsection{Introduction to Cognitive Radio}

The term Cognitive Radio was first published in an article by Joseph Mitola III in 1999, where it was defined as "The point in which wireless personal digital assistants (PDAs) and the related networks are sufficiently computationally intelligent about radio resources and related computer-tocomputer communications to detect user communications needs as a function of use context, and to provide radio resources and wireless services most appropriate to those needs" [8]. He had first proposed the concept a year earlier in a seminar at KTH (the Royal Institute of Technology in Stockholm).

In other words, a CR can be defined as an intelligent wireless communication system that is aware of its surrounding environment i.e. outside world, and uses the methodology of understanding-by-building to learn from the environment and adapt its internal states to statistical variations in the incoming RF stimuli by making corresponding changes in certain operating parameters (e.g., transmit-power, carrier-frequency, and modulation strategy) in real-time [9]. In a CR network, cognitive users (CU) opportunistically use the spectrum allocated to the primary network without causing destructive interference with the PU and vacate the spectrum instantly when the incumbent appears [10].

The experiments conducted by FCC demonstrates that at any given time and location, between $80 \%$ and $90 \%$ of the licensed spectrum remains underutilized. These temporarily unused spectrum slots are called spectrum holes or white spaces, which result in spectral inefficiency [6]. The low spectrum usage in licensed bands coupled with the true scarcity of radio spectrum may eventually hinder the growth of wireless applications. The 
primary features of a $\mathrm{CR}$ transceiver thus include radio environment awareness and spectrum intelligence.

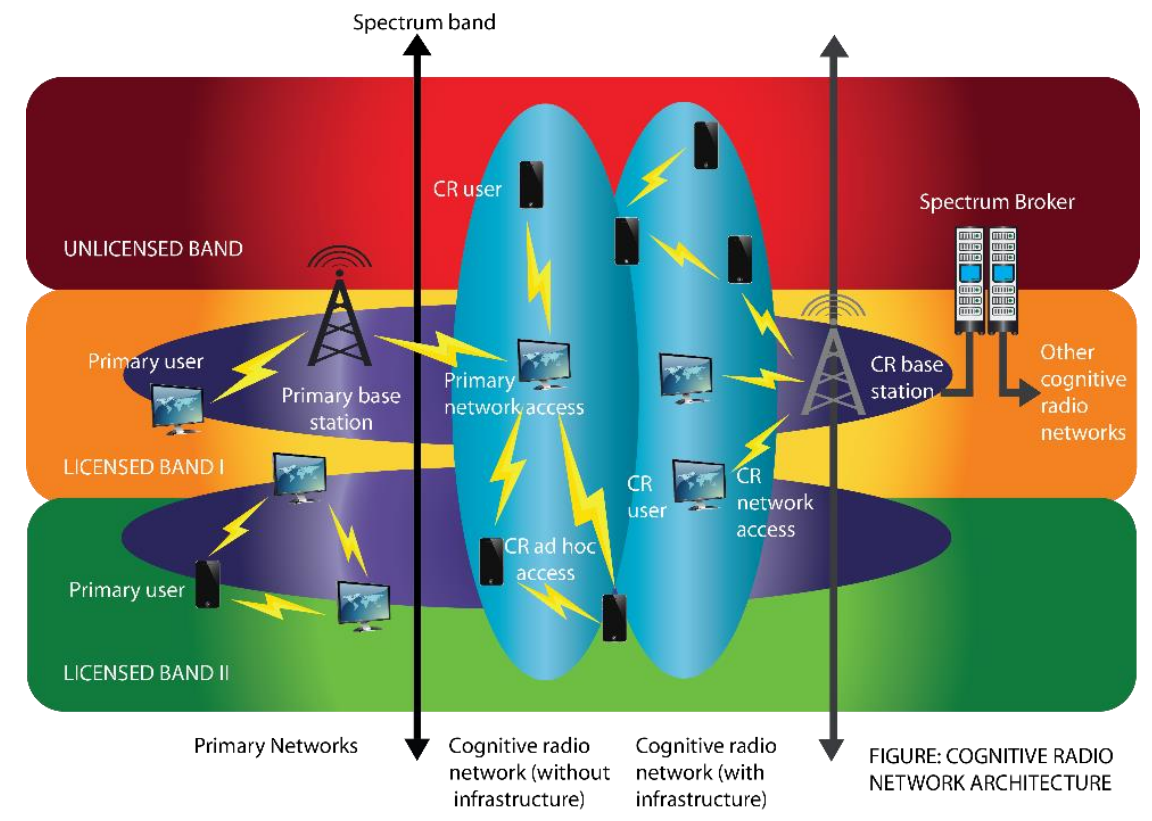

Figure 2: Cognitive Radio Network architecture

\subsubsection{Primary and Secondary Users}

In cognitive radio terminology, primary users (PUs) can be defined as the users who have higher priority or legacy rights on the usage of a specific part of the spectrum. On the other hand, secondary users (SUs), which have lower priority, exploit this spectrum in such a way that they do not cause interference to primary users. Therefore, SUs need to have cognitive radio capabilities, such as sensing the spectrum reliably to check whether it is being used by a PU and to change the radio parameters to exploit the unused part of the spectrum [1]. 


\subsection{Cognitive Radio Features and Potential}

The last few decades have witnessed some of the most astounding levels of advancement in the market for wireless devices and networks, which has furthered an unparalleled evolution. Regulatory agencies in nations all around the globe are now facing the need to allocate chunks of spectrum to different wireless services and applications. Technologies such as Multipleinput multiple-output (MIMO) communications, cooperative communications and heterogeneous networks as elaborated in [6] do enunciate the goal of meeting the rising demand and effective utilization of spectrum. Regardless, spectrum scarcity continues to create challenges for our generation and mounting fear for the next. The need for higher data rates is increasing as an upshot of the transition from voice-only communications to multimedia type applications, as stated in [1]. Given the confines of the natural frequency spectrum, it is evident that the current static frequency allocation schemes simply cannot accommodate the requirements of the growing number of higher data rate devices and applications. The problem aggravates as some frequency bands in the spectrum are heavily used, while others are left unoccupied for the most part, and these potential spectrum holes or white spaces result in the underutilization of available frequency bands. Now is the time for an upsurge of innovative techniques that can offer new ways of sensing and exploiting the available spectrum. CR technologies shall provide exactly that, and spectrum sensing will uncover spectrum for use in dimensions that until now have been unreachable.

IEEE 802.22 is the international standard which uses the CR concept to access the spectrum allocated to TV services (i.e. licensed systems) to provide 
Wireless Regional Area Network (WRAN) services to unlicensed users. Being the first worldwide effort to outline a standardized air interface based on CR techniques for the adaptable use of TV bands on a non-interfering basis, IEEE 802.22 uses tactics like geo-location and spectrum sensing for spectral awareness. According to the recent most ruling by the FCC the CRs operating in the TV broadcast channels, otherwise known as TV Bands Devices, do not necessarily require spectrum sensing. However, the use of spectrum sensing is still not overshadowed because of the handful of advantages it bears over the geo-location database access approach, where a CR must have connection to the internet or any other communication infrastructure, and know its geolocation information before using the database, both of which may not always be accessible. The single point of failure in the database only approach gives rise to security concerns, making it all the more evident that CRs need to be fortified with spectrum sensors [2]. Spectrum sensing is traditionally perceived as the computation of spectral content only, which is the measurement of the RF energy over the spectrum; however, it also involves obtaining the spectrum usage characteristics across multiple dimensions such as frequency, time, geographical space, angle and code [1].

\subsubsection{Cognitive Radio Architecture}

A cognitive user $(\mathrm{CU})$ has the ability to learn the radio's operating environment and adapt to the real-time conditions of its operation, and furthermore, it can independently alter transmission parameters and characteristics for a more flexible, efficient, and comprehensive use of the available spectrum while reducing the risk of harmful interference with the 
PUs [7]. Within the protocol architecture of CR, the RF front-end in the physical layer is implemented based on the SDR transceiver. The adaptive protocols in the MAC, network, transport, and application layers need to be aware of the variations in the $\mathrm{CR}$ environment and should consider the traffic activity of PUs, the transmission requirements of SUs, and variations in channel quality, etc.

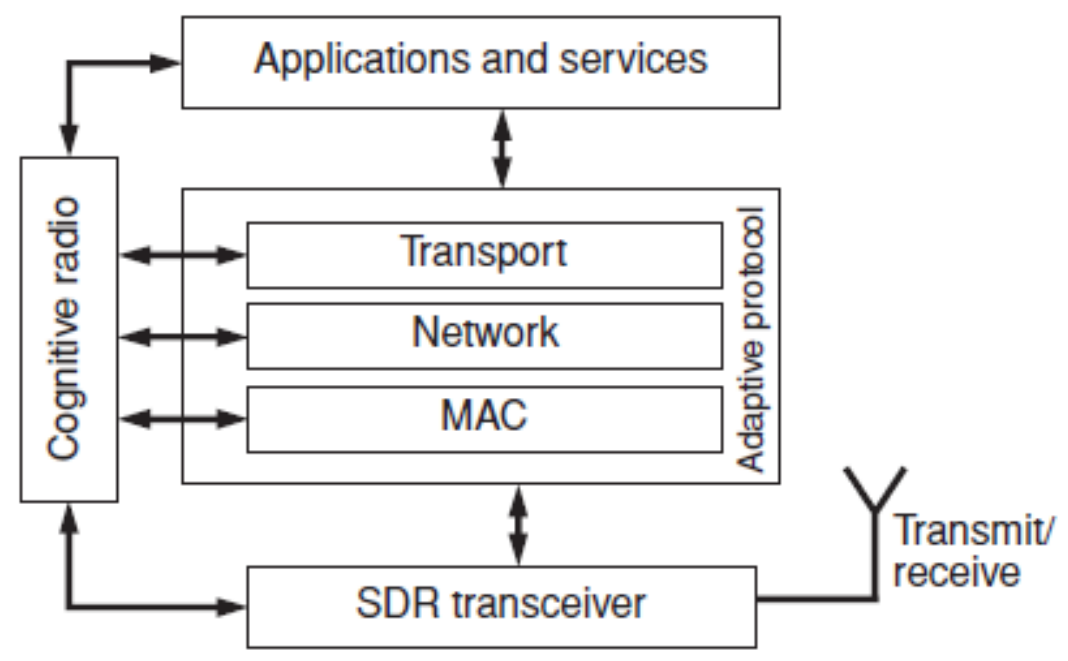

Figure 3: Cognitive Radio protocol stack [3]

To link all modules, a CR control is used to establish interfaces among the SDR transceiver, adaptive protocols, and wireless applications and services. The module uses intelligent algorithms to process the measured signal from the physical layer, and receive information on transmission requirements from the applications to control the protocol parameters in the different layers [3]. 


\subsubsection{Cognitive Radio Interfaces}

A typical radio in today's world interacts with a number of external systems, including the radio user, the network (via a base station), sensors (e.g., spectrum sensor, GPS) and other resources accessible through the network, e.g., other users (social network), informational resources (the Web resources, or more generally the Information Cloud) [11].

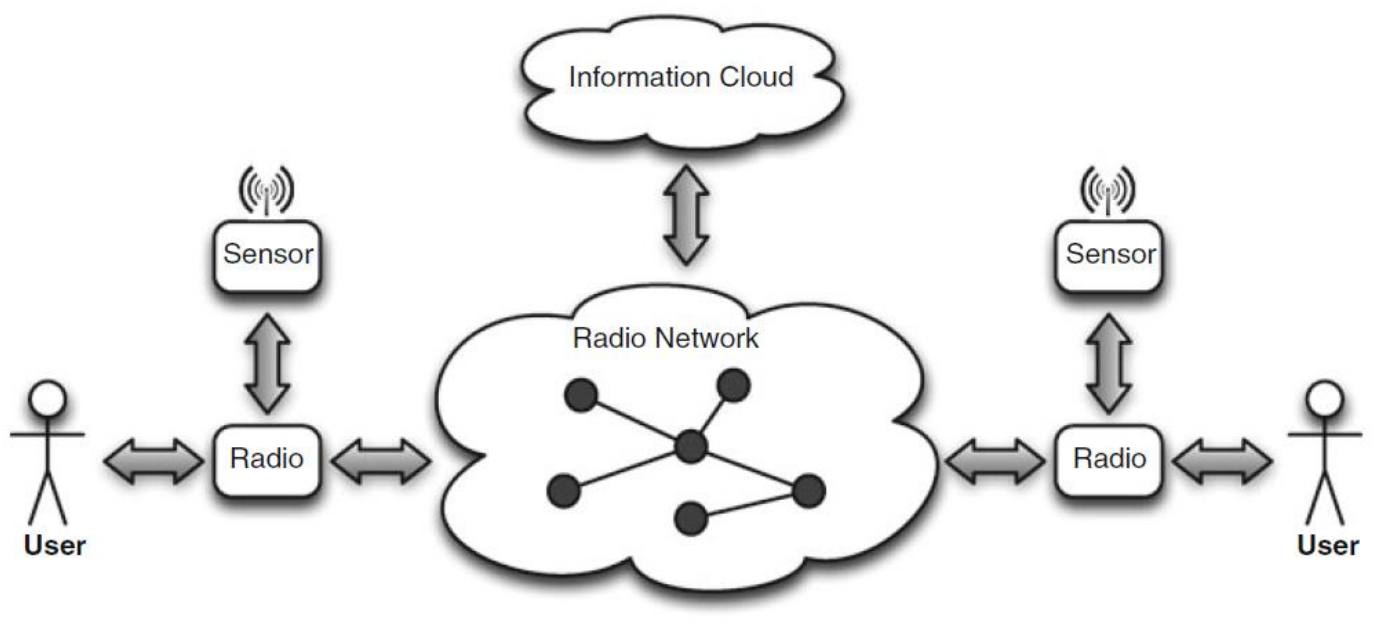

Figure 4: External interfaces of Cognitive Radio [11]

\subsubsection{Dynamic Spectrum Access}

Dynamic spectrum access (DSA) is a new spectrum sharing paradigm that allows SUs to access the abundant spectrum holes or white spaces in the licensed spectrum bands [12]. It has been proposed as an alternative policy to allow the radio spectrum to more efficiently be used, wherein a piece of spectrum can be allocated to one or more PUs. However, the use of that spectrum is not exclusively granted to these users, although they have higher priority in using it. SUs can access the allotted spectrum as long as the PUs 
are not temporally using it, or can share the spectrum with the PUs as long as it can properly be protected. By doing so, the radio spectrum can be reused in an opportunistic manner or shared all the time; thus, the spectrum utilization efficiency can significantly be improved [13].

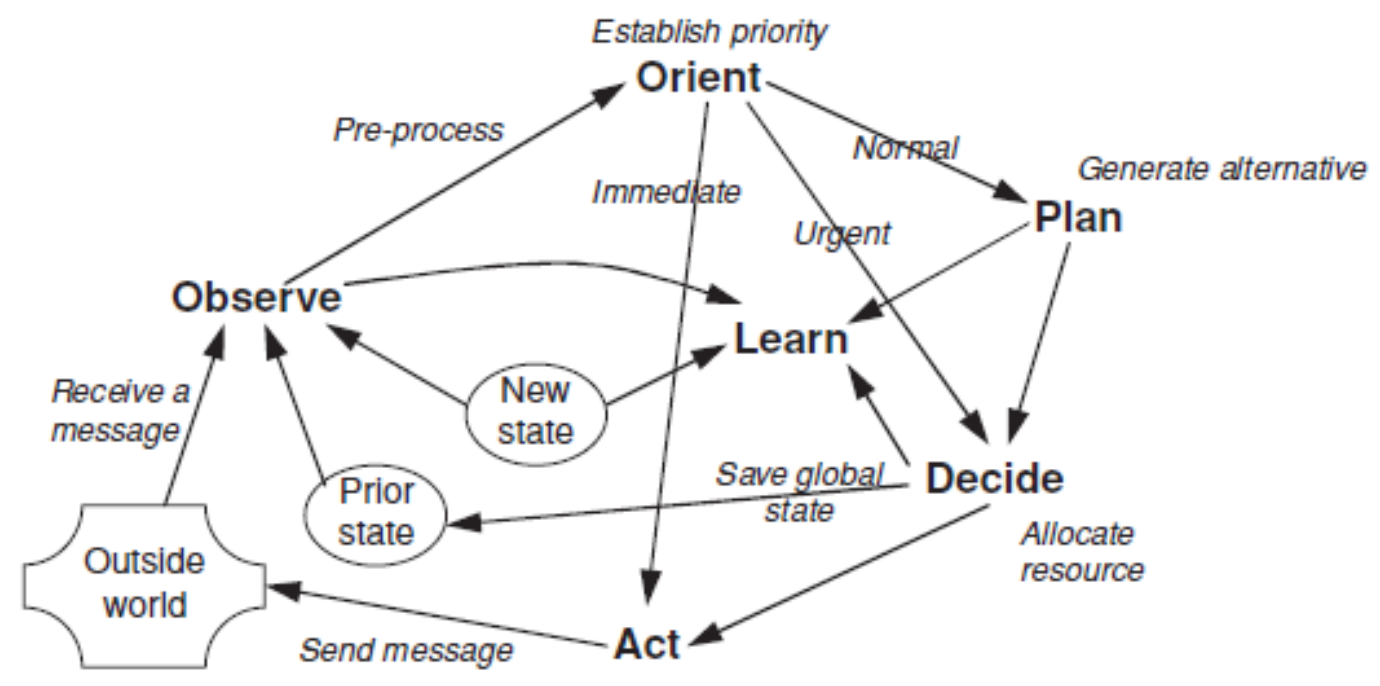

Figure 5: The cognition cycle [14]

\subsubsection{Functions of Cognitive Radio}

Spectrum Sensing: Being the chief component for the establishment of a CR, spectrum sensing is the detection of unused spectrum i.e. spectrum holes or white spaces in the RF bandwidth (which are seen as windows of opportunity by the SUs) as well as the presence and transmissions of the PUs.

Spectrum Management: The management of spectrum is the selection of the best available channels in terms of the strength of the received signal, interference with the PUs, energy efficiency, transmission power, number of users, quality of service, security requirements etc. 
Spectrum Mobility: Spectrum mobility is the upkeep of seamless communication in order to retain adaptability i.e. to let go of a channel when a licensed PU is detected through a spectrum handoff and switch to a new vacant spectrum band.

Spectrum Sharing: While focusing primarily on power allocation, spectrum sharing is the coordination of access to a spectrum band by a number of users.

\subsubsection{Cognitive Radio Components}

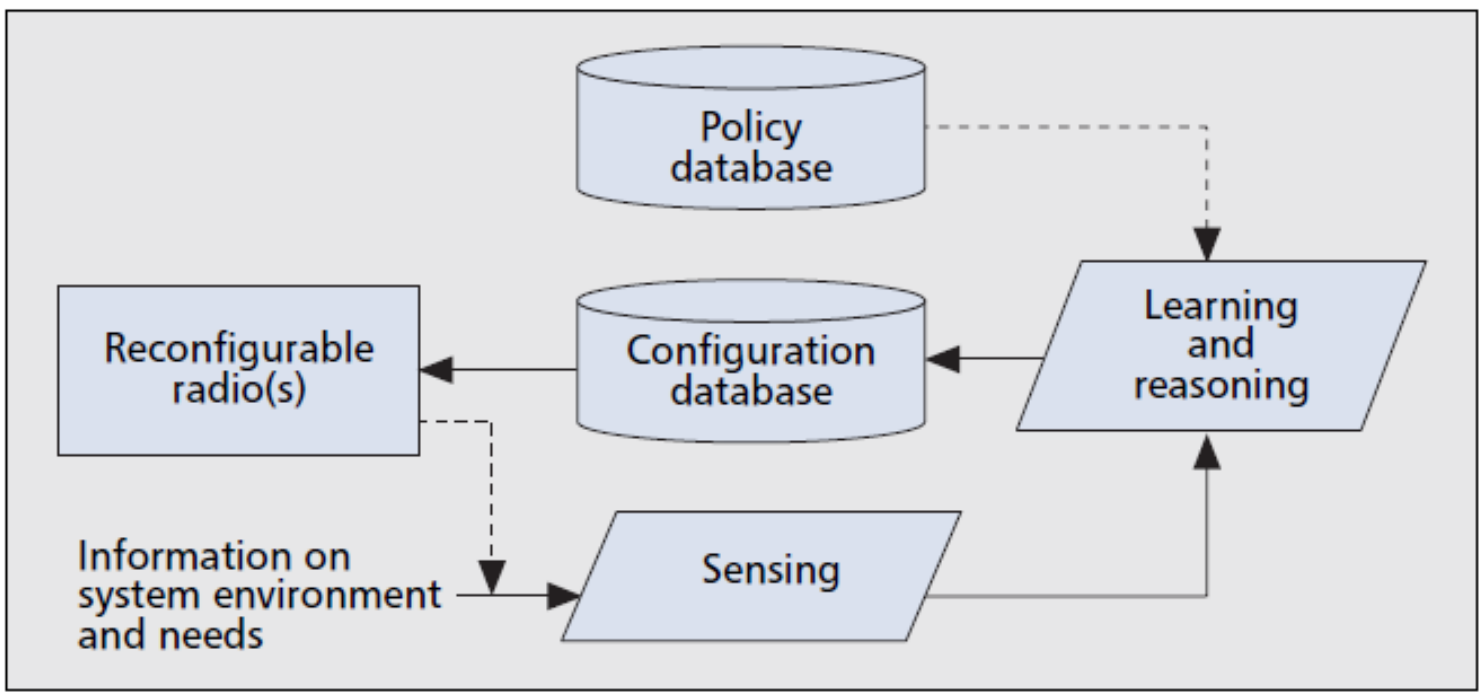

Figure 6: Components in a Cognitive Radio System [15]

The three approaches to identifying spectrum opportunities include database registry, beacon signals, and spectrum sensing. Therefore, there must be at least one reconfigurable radio component with multiple parameters, including operating frequency and bandwidth. A sensing engine will be able to accept inputs from either this radio component, or other sources including networked nodes, data sources on the Internet and geo-location data. The 
system can have a policy database that determines what behavior is acceptable in what circumstances, which can be dynamically configurable allowing for policy changes. The system must have a reasoning engine that accepts inputs from the sensing engine and the policy database, which is capable of learning, based on experience. Finally, a configuration database would maintain the current configuration of the radio components. A simple CR system might have a single reconfigurable radio component accepting sensing information from a single local node and no external data sources. [15].

\subsection{Spectrum Sensing in Cognitive Radio}

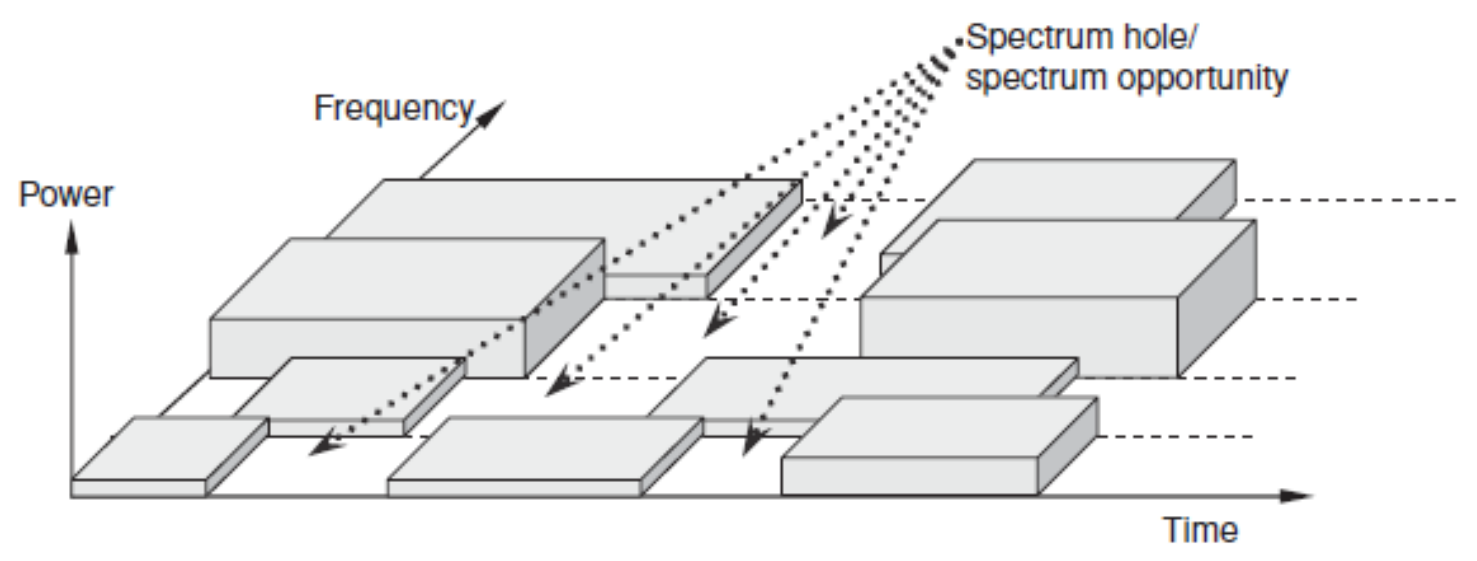

Figure 7: Spectrum hole (or spectrum opportunity) [3]

A CR transceiver detects a vacant spectrum in terms of band, location, and time and presumes the method of accessing it without interfering with the transmission of the licensed user. As defined in [9], a spectrum hole is a band of frequencies assigned to a PU, but, at a particular time and specific geographic location, the band is not being utilized by that user, which is a gold 
mine for CRs. As soon as a CR starts to utilize a white space, it no longer has a choice regarding the sensing mode and has to sense the band proactively at periodic intervals. This will ensure timely detection of any PUs trying to reclaim the band, as mandated by the regulatory bodies [16], keeping in mind that avoiding interference with potential PUs in the vicinity is one of the most fundamental requirements of a CR. Primary user networks on the other hand, are not required to change PU infrastructure for spectrum sharing with cognitive networks. Sensing schemes of different sensitivities and rates are required for detecting different classes of PU signals, for instance, it is much easier to detect TV broadcast signals over GPS signals [17].

As for the cross-layer functionalities of a CR, the physical layer carries out sensing, cognition (PU detection: energy and footprint) and adaptation (optimization of spectrum usage: power band and modulation), unlike the MAC layer which combines sensing measurements and jointly allocates spectrum. The various kinds of spectrum sensing in the physical layer are shown below in Figure 8.

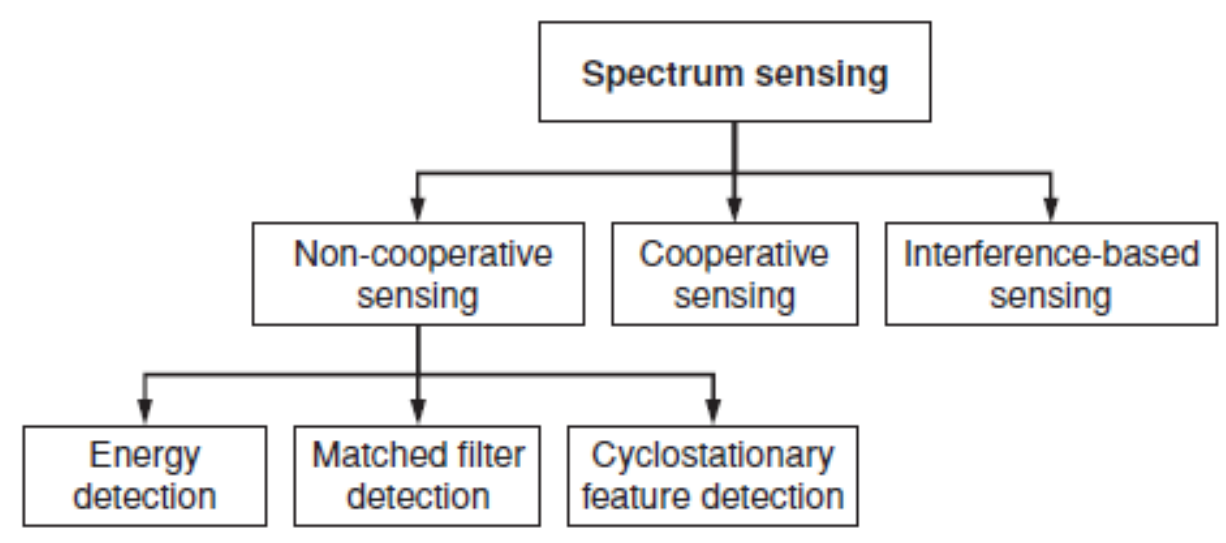

Figure 8: Types of Spectrum Sensing [3] 


\subsubsection{Non-Cooperative Spectrum Sensing}

Non-cooperative spectrum sensing is used by an unlicensed SU to detect the transmitted signal from a licensed user by using local measurements and local observations. Notable non-cooperative sensing algorithms include matched filter detection, energy detection and cyclostationary feature detection, the advantages and disadvantages are mentioned in Table 2. Techniques requiring prior knowledge about the PU's signal for comparing particular signal features to the SU's received signal are called coherent signal detection techniques, e.g. matched filter. Non-coherent detection techniques compare the received signal to a threshold defined on the basis of features that are independent of primary signal knowledge, e.g. energy detection [18].

Table 2: Summary of the Non-Cooperative Spectrum Sensing techniques [3]

\begin{tabular}{|c|c|c|}
\hline Sensing Algorithm & Advantages & Disadvantages \\
\hline Energy Detection & $\begin{array}{c}\text { No prior information } \\
\text { required } \\
\text { and low cost }\end{array}$ & $\begin{array}{c}\text { Cannot work in low SNR; } \\
\text { cannot } \\
\text { distinguish primary and } \\
\text { other } \\
\text { secondary users }\end{array}$ \\
\hline Matched Filter & $\begin{array}{c}\text { Optimal performance } \\
\text { and low } \\
\text { cost }\end{array}$ & $\begin{array}{c}\text { Prior knowledge of } \\
\text { primary user's } \\
\text { signal is required }\end{array}$ \\
\hline Fyclostationary & $\begin{array}{c}\text { Robust in low SNR } \\
\text { and } \\
\text { interference }\end{array}$ & $\begin{array}{c}\text { Partial information of } \\
\text { primary } \\
\text { user; high computation } \\
\text { cost }\end{array}$ \\
& & \\
\hline
\end{tabular}




\subsubsection{Cooperative Spectrum Sensing}

The main idea of cooperative sensing is to enhance the sensing performance by exploiting the spatial diversity in the observations of spatially located CR users. CR users can share their sensing information via cooperation in order to make a combined decision, which is irrefutably more accurate than individual decisions [19]. There are broadly two approaches to cooperative spectrum sensing, namely, the centralized approach and the distributed approach.

\subsubsection{Interference-Based Spectrum Sensing}

In interference-based sensing, the sensing algorithm measures the noise/interference level from all sources of signals at the receiver of the licensed user. This information is used by an unlicensed user to control the spectrum access without violating the interference temperature limit. Alternatively, an unlicensed transmitter may observe the feedback signal from a licensed receiver to gain knowledge on the interference level [3].

\subsubsection{Channel Sensing Hypothesis}

Statistical hypothesis testing is archetypally performed in spectrum sensing, in order to test the sensing results for a binary decision on the presence of PUs. It is a classical hypothesis testing approach, where $\mathrm{H}_{0}$ is the null hypothesis, which states that there is no primary signal in a certain band, and $\mathrm{H}_{1}$ is the alternative hypothesis, which confirms the presence of the PU in that frequency band. A test statistic is compared with a specific threshold to 
discriminate between the two hypotheses, and system performance is evaluated in terms of probability of detection $P_{d}$ and probability of false alarm $\mathrm{P}_{\mathrm{f}}$. The model for signal detection at time $\mathrm{t}$ can be described as [4]:

$$
x(t)=\left\{\begin{array}{rr}
n(t), & H_{0} \\
h * s(t)+n(t), & H_{1}
\end{array}\right.
$$

where $\mathrm{x}(\mathrm{t})$ is the received signal at an unlicensed $\mathrm{SU}, \mathrm{h}$ is the channel gain, $\mathrm{s}(\mathrm{t})$ is the transmitted signal of the licensed PU which is presumed to be a Gaussian random process with variance $\sigma_{\mathrm{s}}{ }^{2}$, and $\mathrm{n}(\mathrm{t})$ is the Additive White Gaussian Noise (AWGN), which is a basic noise model used in information theory to mimic the effect of many random processes that occur in nature, having a zero-mean and variance $\sigma_{\mathrm{n}}{ }^{2}$. The performance of a spectrum sensing technique is generally measured in terms of the following metrics that are expressed in terms of mathematical probability [3]:

Probability of detection, $\mathrm{P}_{\mathrm{d}}=$ Prob $\left\{\right.$ decision $\left.=\mathrm{H}_{1} \mid \mathrm{H}_{1}\right\}$

Probability of false alarm, $\mathrm{P}_{\mathrm{f}}=$ Prob $\left\{\right.$ decision $\left.=\mathrm{H}_{1} \mid \mathrm{H}_{0}\right\}$

Probability of missed detection, $\mathrm{P}_{\mathrm{m}}=$ Prob $\left\{\right.$ decision $\left.=\mathrm{H}_{0} \mid \mathrm{H}_{1}\right\}$

Table 3: Channel Sensing Hypothesis in Spectrum Sensing [3]

\begin{tabular}{|c|c|c|c|}
\hline \multicolumn{2}{|c|}{} & \multicolumn{2}{c|}{ SU Response (Decision / Hypothesis) } \\
\cline { 3 - 4 } \multicolumn{2}{|c|}{} & PU Present & PU Absent \\
\hline \multirow{2}{*}{$\begin{array}{c}\text { PU } \\
\text { Signal }\end{array}$} & Present & Correct detection $\left(\mathrm{P}_{\mathrm{d}}\right)$ & Missed detection $\left(\mathrm{P}_{\mathrm{m}}=1-\mathrm{P}_{\mathrm{d}}\right)$ \\
\cline { 2 - 4 } & Absent & False alarm $\left(\mathrm{P}_{\mathrm{f}}\right)$ & Correct rejection \\
\hline
\end{tabular}




\section{CHAPTER 3}

\section{Enhanced Energy Detection Using Matched Filter}

The detection of a signal in the presence of noise requires processing which is governed by the characteristics of the noise and the signal; when the noise is Gaussian and the signal has a known form, the appropriate processing technique is a matched filter or its correlator equivalent. When the signal has an unknown form, however, it is appropriate to consider the signal as a sample function of a random process. When the signal statistics are known, this knowledge can often be used to design suitable detectors [20].

\subsection{Conventional Energy Detection Algorithm}

The energy detector is built around the principle that the transmitted signal will only be perceived by comparing the output of the energy detector against a threshold which depends on the signal and noise variance. The noise variance is an explicit parameter for the design of the energy detector; all existing works assume a perfect knowledge of the noise level at the receiver, which allows for an appropriate threshold design. In this case the Energy detector works with arbitrarily small values of probability of false alarm $\left(\mathrm{P}_{\mathrm{f}}\right)$ by using a sufficiently large observation time, $t$, signals received with low SNR values, and a predefined suitable threshold energy level under Constant False Alarm Rate (CFAR). Signal-to-noise ratio (SNR) generically refers to the dimensionless ratio of the desired signal to the level of background noise, it parameterizes the performance of optimal signal processing systems when the noise is Gaussian [21]. In practical radar signal detection systems, 
detecting a target automatically in a non-stationary noise and clutter (unwanted echoes in electronic systems) background while maintaining a constant probability of false alarm is difficult. Constant False Alarm Rate (CFAR) detection, which refers to a common form of adaptive algorithms used in radar systems to detect target returns against a background of noise, clutter and interference, is the key to solving this problem [22]. The two chief metrics governing the detection performance phenomenon of an algorithm for spectrum sensing are as follows:

Probability of detection $\left(\mathrm{P}_{\mathrm{d}}\right)$ : A CR has detected the presence of a PU when the spectrum band is occupied i.e. correct detection of the signal.

Probability of false alarm $\left(\mathrm{P}_{\mathrm{f}}\right)$ : A CR has detected the presence of a PU when the spectrum band is in fact free i.e. incorrect detection of the signal.

The sensing decision can be formulated into a binary hypothesis as given in equation (1). $\mathrm{H}_{0}$ and $\mathrm{H}_{1}$ are the hypotheses 0 and 1 which denote that the PU is absent and present, respectively.

After passing through a noise pre-filter or a Band Pass Filter (BPF) over a bandwidth, the received signal is denoted as $x(t)(t=1,2, \ldots, N), N$ being the number of samples used in the sensing algorithm. Here, $n(t)$ represents the Additive White Gaussian noise (AWGN), assumed to be independent and identically distributed with zero-mean and variance $\sigma_{n}^{2}$; $s(n)$ is the PU signal, also assumed to be an independent and identically distributed random process with zero-mean and variance of $\sigma^{2}$, and $h$ is the channel gain. With the signal and noise variance, the SNR for random variables can be defined as: [21]

$$
S N R=\frac{\sigma_{s}^{2}}{\sigma_{n}^{2}}
$$


When there are sufficient sample points $\mathrm{N}$, the decision threshold can be derived for a target $\mathrm{P}_{\mathrm{d}}$ or $\mathrm{P}_{\mathrm{f}}$. Under hypothesis $\mathrm{H}_{1}$, the threshold $\lambda_{\mathrm{Pd}}$ can be set for a constant detection rate (CDR) as the following: [5]

$$
\lambda_{P_{d}}=\left(\sigma_{n}^{2}+\sigma_{s}^{2}\right)\left(1+\frac{Q^{-1}\left(P_{d}\right)}{\sqrt{N}}\right)
$$

Similarly, under hypothesis $\mathrm{H}_{0}$, the threshold $\lambda_{\mathrm{Pf}}$ can be set for Constant False Alarm Rate (CFAR) as the following: [5]

$$
\lambda_{P_{f}}=\sigma_{n}^{2}\left(1+\frac{Q^{-1}\left(P_{f}\right)}{\sqrt{N}}\right)
$$

The threshold based on CFAR is commonly applied in conventional energy detection algorithms; hence, we have employed the equation (4) in our formulated algorithm.

\subsection{Design of our Algorithm}

Matched filter detection is appropriate when the transmission of a licensed user has pilot, preambles, and synchronization word or spreading codes, which can be used to create the template for spectrum sensing [3]. Once the matched filter template is created in the network simulator, as described in the segment 3.5 'Simulation Setup using (OMATLAB 2017a', and a fixed SNR value is set, one can proceed with the steps in our devised algorithm. The SNR expressed in a logarithmic scale in decibels $(\mathrm{dB})$ can be calculated once the ratio of the signal variance and the noise variance is determined, using the formula in [21]: 


$$
S N R=10 \log _{10} \frac{\sigma_{s}^{2}}{\sigma_{n}^{2}}
$$

Since we are implementing Gaussian signals, we assumed for our sensing algorithm, a noise variance with zero-mean and variance 1, i.e. $\sigma_{\mathrm{n}}{ }^{2}=1$. Substituting this value into (5) the value of the signal variance can be attained, which is used to construct the transmitted signal of the PU for our algorithm. The formula for the signal variance, as expressed in equation (6), is therefore:

$$
\sigma_{s}^{2}=10^{\left(\frac{S N R}{10}\right)}
$$

We fixed the value of $\mathrm{P}_{\mathrm{f}}$ to a range of 0 to 1 , and considered a minimal value of 100 samples for our sample size $N$, which means $P_{f}$ that will have at least a minimum of 100 values set in between 0 and 1 . Since we implemented an energy threshold under Constant False Alarm Rate, (keeping in mind that Q-function is the tail distribution function of the standard normal distribution) after incorporating the value of noise variance in the equation given by Dr. Y. C. Liang [5], we derived the formula of energy threshold, as follows:

$$
\lambda=\left(\frac{Q^{-1}\left(P_{f}\right)}{\sqrt{N}}+1\right)
$$

Following the determination of the energy threshold, we considered 10,000 Monte Carlo simulations in order to achieve our desired output. However, if the number of Monte Carlo simulations goes higher, the randomness will decrease and the ROC curves will become smoother. In each iteration we generated a noise vector of sample size $\mathrm{N}$, which has a zero-mean and a variance of 1, using the built-in function for generating random values in CMATLAB. This was done to create the Additive White Gaussian Noise 
(AWGN) for the background in our simulation environment. A real valued Gaussian signal was generated to be the PU signal by carrying out the product of the square-root of signal variance achieved from equation (6), and another vector of random values for the additive stochastic noise within the transmitted signal. We then added the signal and the AWGN together to simulate a realistic received signal at the SU.

Moving onto the crucial part of our algorithm, where we crosscorrelated the received signals with the matched filter template of the PU signal previously generated through built-in functions of CMATLAB 2017a, the parts that were matched with the received signal samples at the SU, were maximized. We used these resulting signals, considered absolute values and mathematically squared them, just like the squaring device in a traditional energy detector does. We then used these values to determine the energy received from the signal after integrating the squared value over a substantially large period of time. Monte Carlo methods provide a way out of complicated exponential increase in computation time, which was greatly useful for our algorithm. By the central limit theorem, this method displays $1 / \sqrt{ } \mathrm{N}$ convergence, and that is what we have used in finding the signal energy. For variance reduction, we used importance sampling in the Monte Carlo Method, as elaborated in [23].

$$
E=\int_{-\infty}^{\infty}|x(t)|^{2} d t
$$

This received energy, E, in equation (8) was used as the test statistic for our algorithm, and we therefore compared it with the energy threshold we obtained in equation (7); the results were stored for all of the 100 samples with 
accordance to the Binary hypothesis. We divided the number of detections (where the signal energy, E was greater than the energy threshold, $\lambda$ ) by the number of Monte Carlo simulations, in order to find the $\mathrm{P}_{\mathrm{d}}$. We obtained the probability of missed detection $\left(\mathrm{P}_{\mathrm{m}}\right)$ by subtracting the $\mathrm{P}_{\mathrm{d}}$ from 1 . We then found the ROC curves with the resulting values of $\mathrm{P}_{\mathrm{d}}$ and $\mathrm{P}_{\mathrm{f}}$.

\subsubsection{Block Diagram}

The block diagram for our proposed sensing algorithm as defined in Figure 9 has been created following the footsteps of the conventional energy detector.

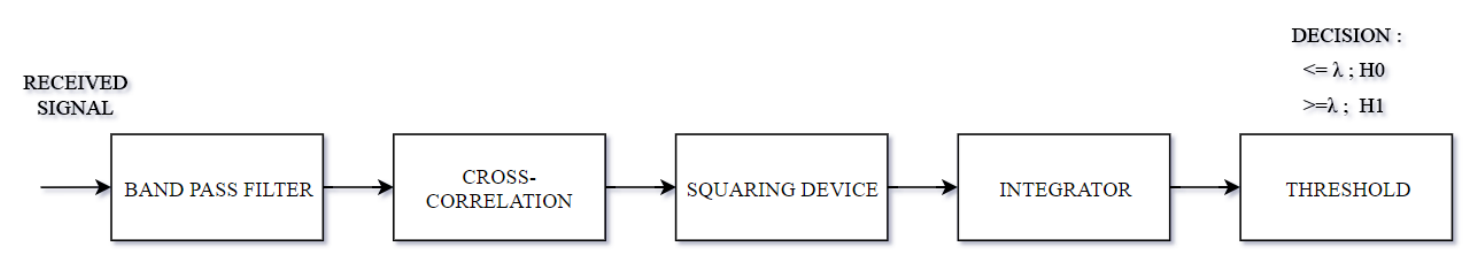

Figure 9: Block diagram for Enhanced Energy Detection Algorithm using Matched Filter

The received signal at the SU first passes through a Band Pass Filter $(\mathrm{BPF})$, which is deployed to isolate or filter out certain frequencies that lie within a particular band or range of frequencies. Following this, the matched filter template of the PU signal is determined, and then cross-correlation is carried out between the two signals. After this stage, the final received signal is taken, and passed through a squaring device and an integrator. Finally, the signal energy is compared with the energy threshold obtained through operation under CFAR. 


\subsubsection{Flowchart}

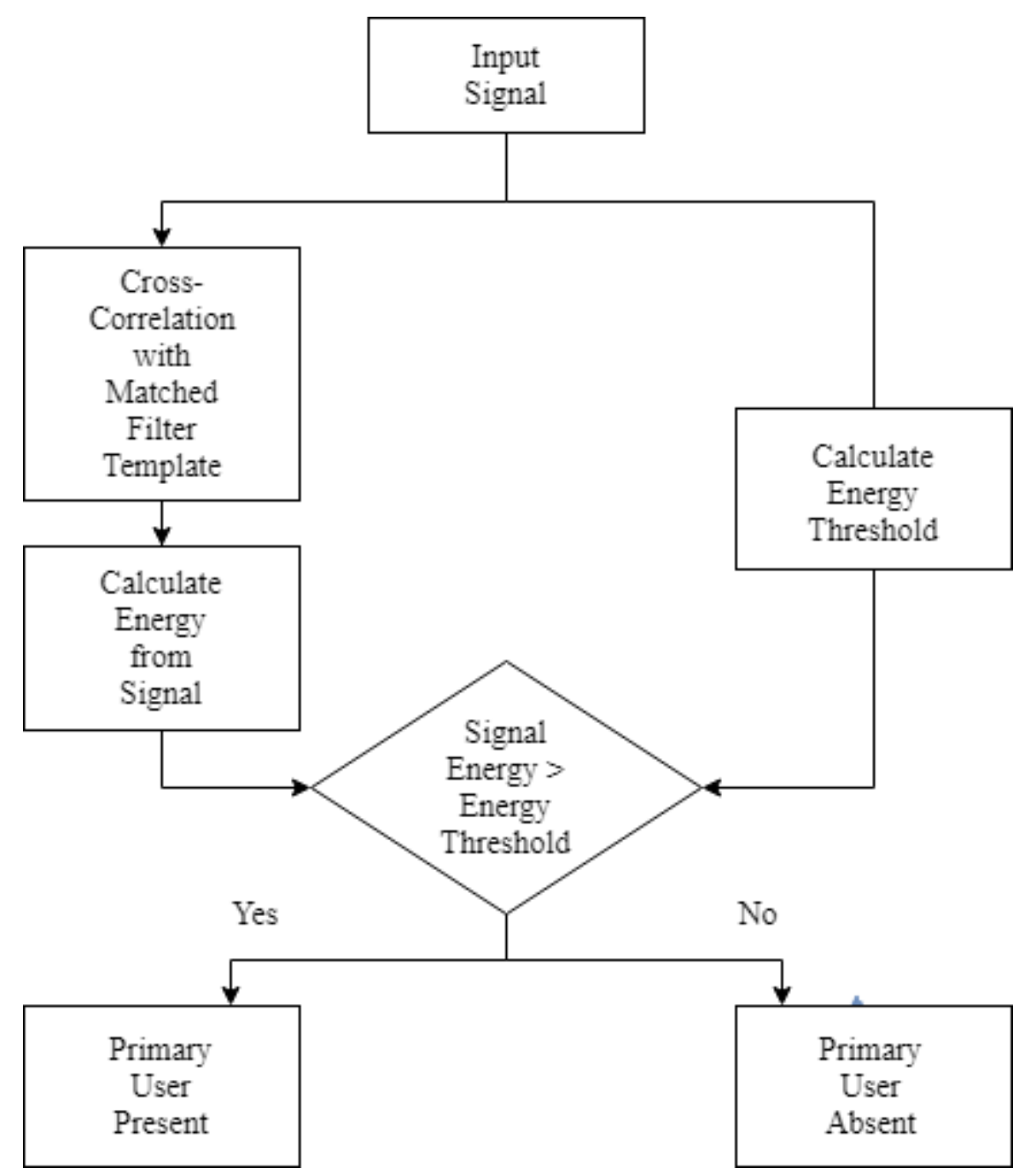

Figure 10: Flowchart for Enhanced Energy Detection Algorithm using Matched Filter

The flowchart of our proposed model is presented in Figure 10 which describes how the final deductions regarding the channel occupancy are made - if the calculated energy of the signal received at the $\mathrm{SU}$ is greater than that of the threshold, it can be said that the PU is present. If the calculated energy of the signal received at the $\mathrm{SU}$ is less than that of the threshold, it can be inferred that the PU is absent, and a spectrum hole or white space is available. 


\subsection{Pseudo-code}

The pseudo code for our proposed algorithm is given below, the design of which has been previously explained.

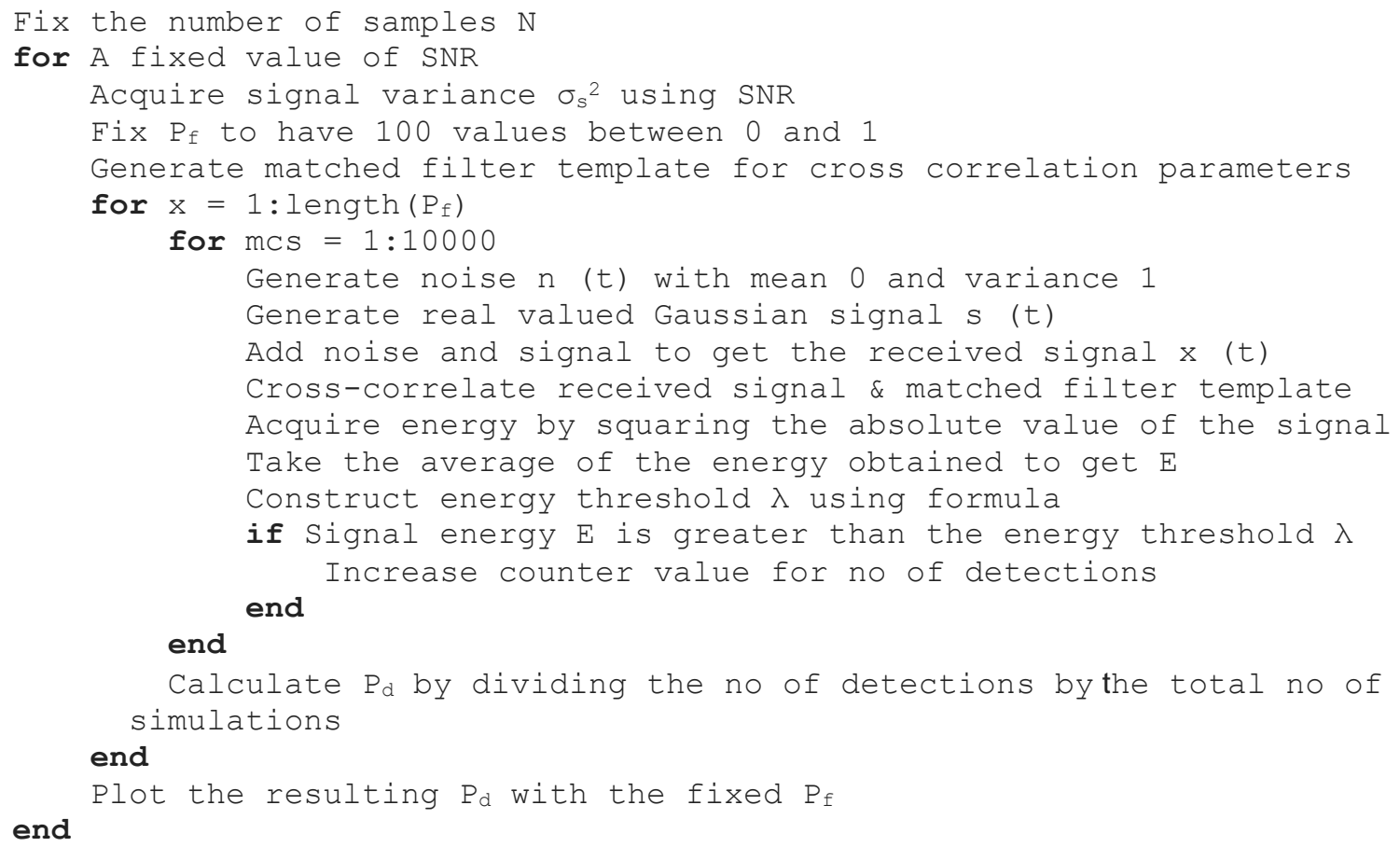

\subsection{Simulation Setup using OMATLAB 2017a}

We used Phased Array System Toolbox ${ }^{\text {TM }}$ in CMATLAB 2017a, specifically the LinearFMWaveform object from the package 'phased' which creates a linear FM pulse waveform. After generating the step response, we implemented the getMatchedFilter function to obtain the matched filter coefficients for waveform. Lastly, we used phased.MatchedFilter to create the object which we then passed through the step function one more time to generate the final template. We used the built-in random function to generate 
the AWGN, to be the background noise in our environment. We used the same function to generate the additive stochastic noise in the real valued Gaussian primary signal that was transmitted by the PU. In order to do a crosscorrelation between the PU signal template and the received signal at the SU, we used the built-in cross-correlation function, and proceeded with the detection algorithm. 


\section{CHAPTER 4}

\section{Results and Evaluation}

This segment contains the results analysis and evaluation of our devised algorithm, the results obtained from implementing the enhanced energy detection algorithm using matched filter in CMATLAB 2017a generated the following figures.

\subsection{Signal Construction}

Figure 11 shows the construction of the received signal at the SU using the real valued Gaussian PU signal and the Additive White Gaussian Noise.

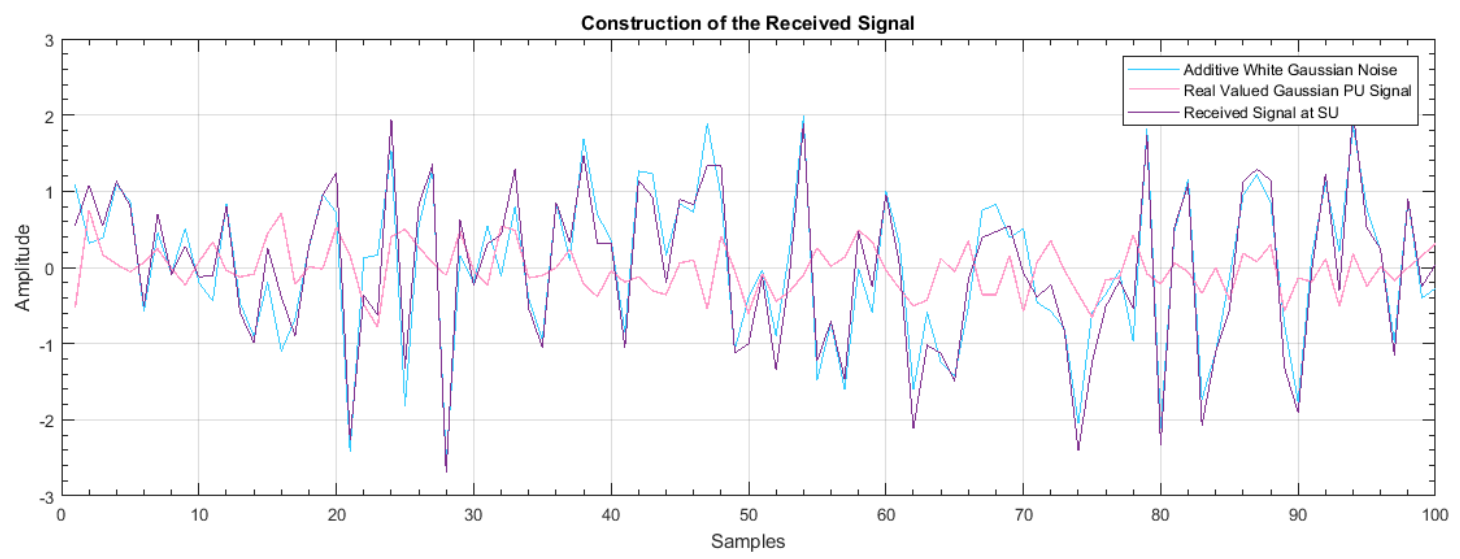

Figure 11: Construction of the Received Signal

\subsection{Cross-correlation of Signal and Matched Filter Template}

In signal processing, cross-correlation is a measure of similarity of two series as a function of the displacement of one with respect to the other, where the parts which are almost identical and in phase with one another are 
maximized. This is also known as a sliding dot product or sliding innerproduct. In our algorithm, we used the received signal at the SU and the matched filter template to carry out cross-correlation.

The SU using matched filter, in actuality, is receiving the correlation function of the input signal and the desired PU signal. When there are several communicators using the same channel without phase coherence, it is desirable that the cross-correlation functions be small in absolute value [24]. Figure 12 shows the difference between a received signal before and after cross-correlation.

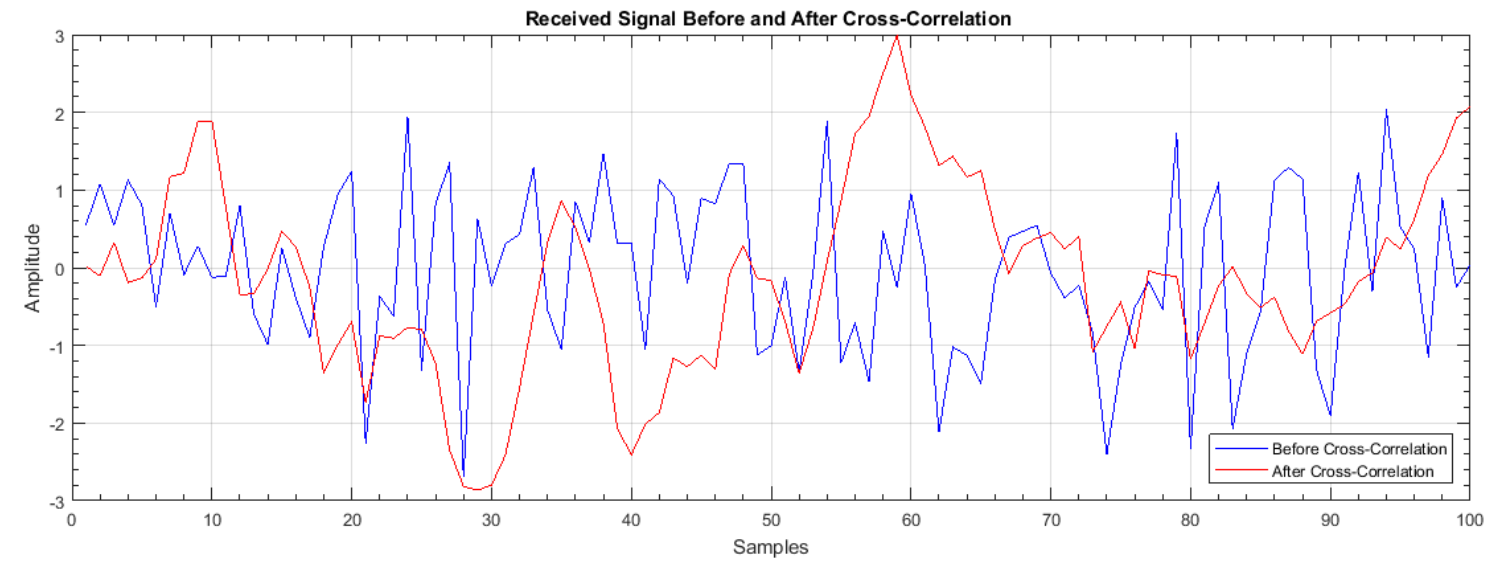

Figure 12: Received Signal before and after Cross-correlation 


\subsection{Signal Energy and Energy Threshold}

Figure 13 shows the energy comparison for the conventional and the enhanced energy detection algorithms with the energy threshold.

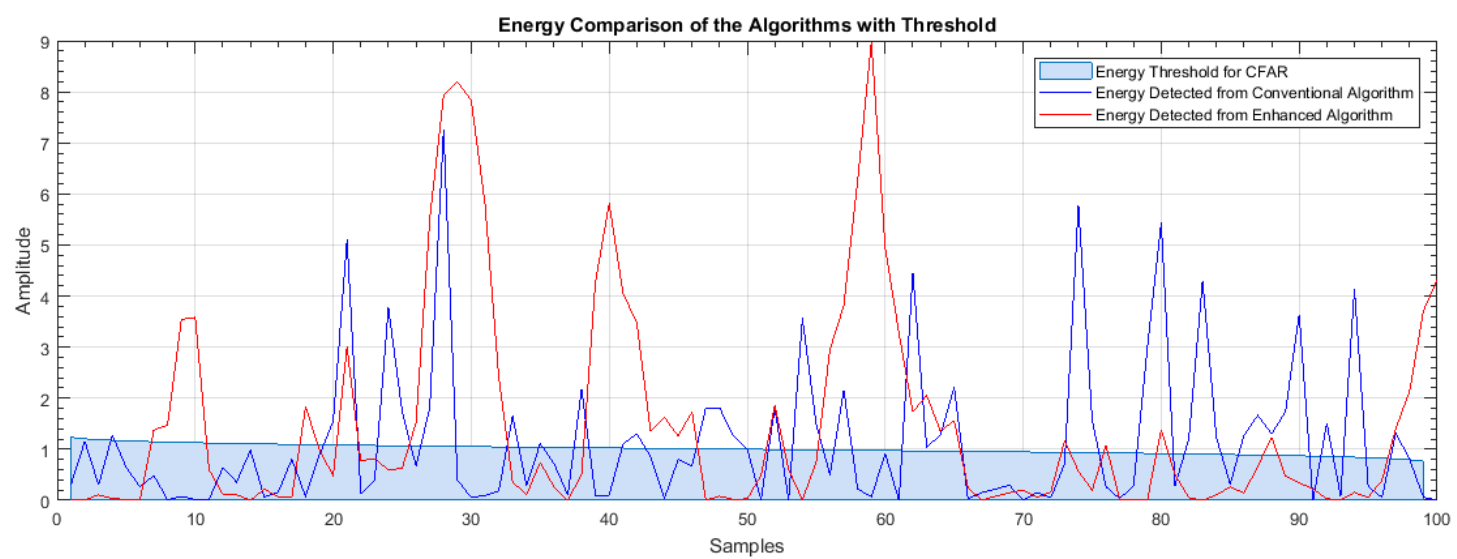

Figure 13: Energy Comparison of the Algorithms with Threshold

Here, the red solid line represents the signal energy detected from the enhanced energy detection algorithm, and the blue solid line represents the signal energy from the conventional energy detection algorithm. The light blue area in the graph represents the energy threshold under Constant False Alarm Rate (CFAR), with which we made appropriate comparisons following the channel sensing hypothesis. 


\section{4 $P_{d}$ vs. $P_{f}$ of the Algorithms at a Fixed SNR Value}

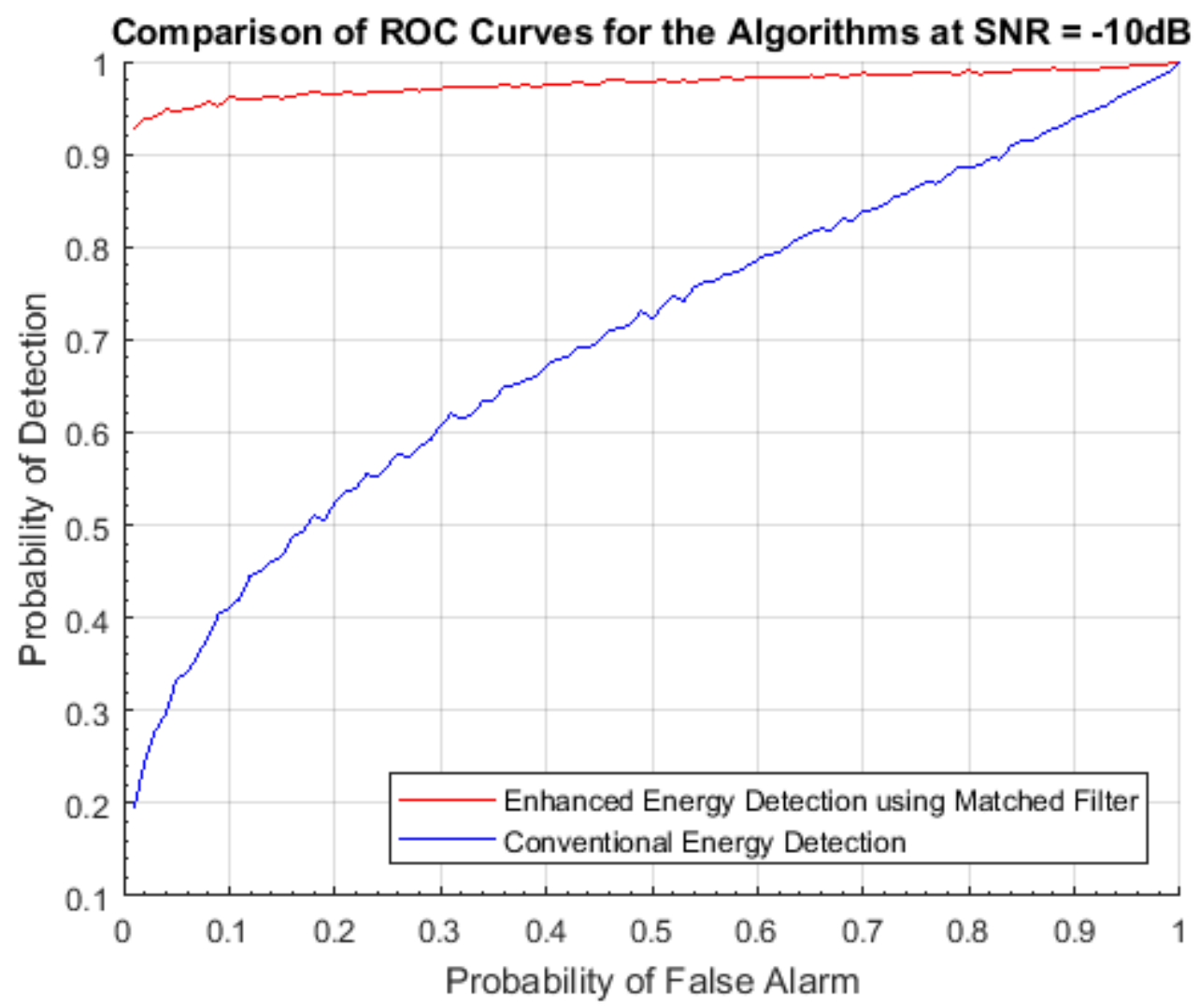

Figure 14: $\mathrm{P}_{\mathrm{d}}$ vs. $\mathrm{P}_{\mathrm{f}}$ for the Algorithms at a Fixed SNR

If we compare the two algorithms at a fixed SNR value of $-10 \mathrm{~dB}$ as in

Figure 14, it is evident that the enhanced energy detection algorithm using matched filter outperforms the conventional algorithm of energy detection. According to the spectrum sensing requirements set by the standard IEEE 802.22 for detection accuracy, the specifications on probability of false alarm and probability of detection are [25],

$$
\begin{aligned}
& \mathrm{P}_{\mathrm{f}} \leq 0.1 \\
& \mathrm{P}_{\mathrm{d}} \geq 0.9
\end{aligned}
$$


While considering $\mathrm{P}_{\mathrm{f}}=0.1$, the $\mathrm{P}_{\mathrm{d}}$ for the conventional algorithm is approximately 0.42 , whereas the $\mathrm{P}_{\mathrm{d}}$ for the enhanced algorithm is approximately 0.96 . The simulation results show that our proposed algorithm is justified in accordance to the IEEE 802.22 requirements, and it can considerably improve the system performance of a conventional energy detector.

\section{5 $P_{m}$ vs. $P_{f}$ of the Algorithms at a Fixed SNR Value}

Figure 15 is a plot of probability of missed detection $\left(\mathrm{P}_{\mathrm{m}}\right)$ vs. probability of false alarm $\left(\mathrm{P}_{\mathrm{f}}\right)$. The value of $\mathrm{P}_{\mathrm{m}}$ for the enhanced algorithm is substantially lower than that of the conventional algorithm, which is satisfactory.

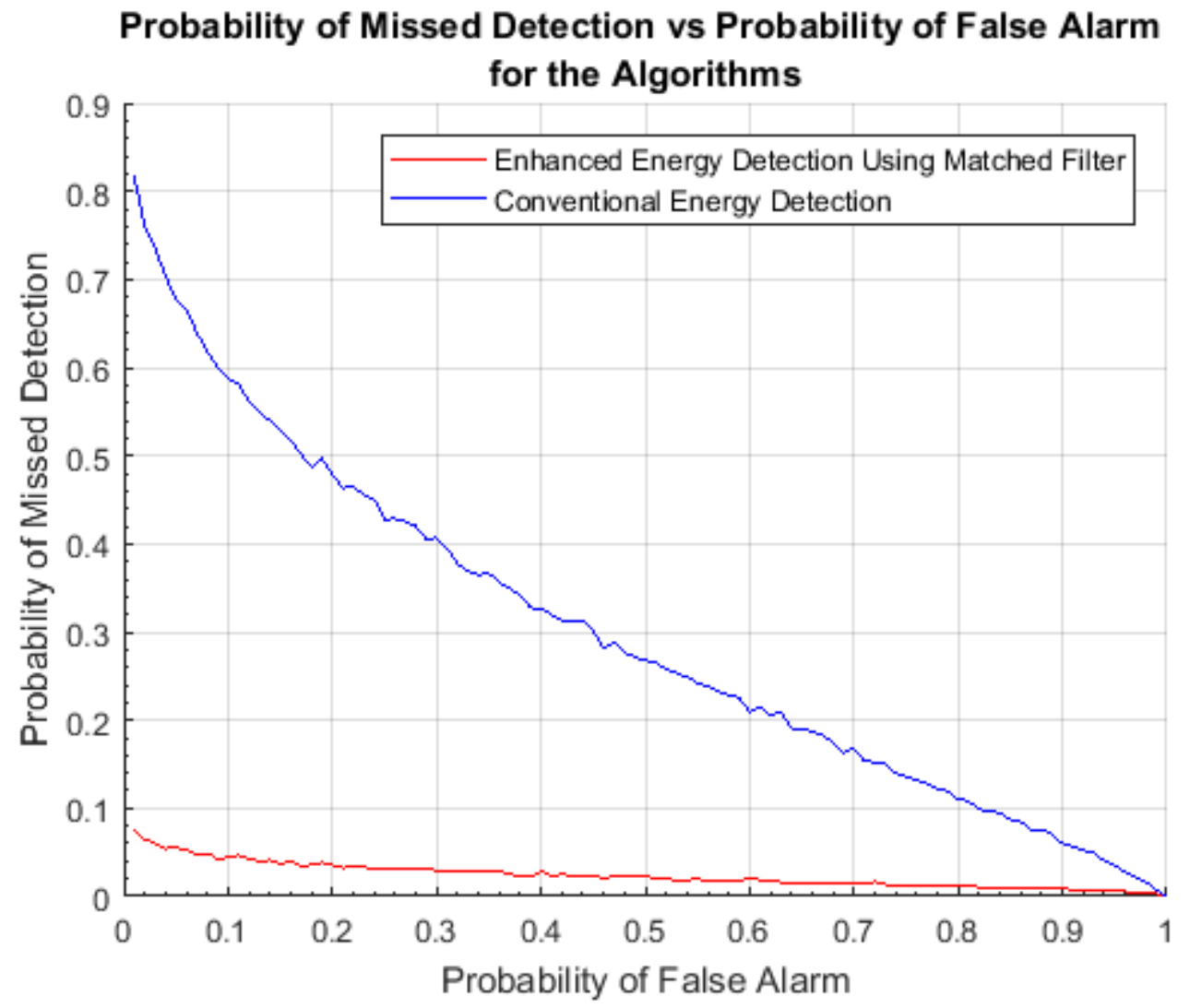

Figure 15: $\mathrm{P}_{\mathrm{m}}$ vs. $\mathrm{P}_{\mathrm{f}}$ for the Algorithms at a Fixed SNR 


\subsection{ROC Curves for a Range of SNR Values}

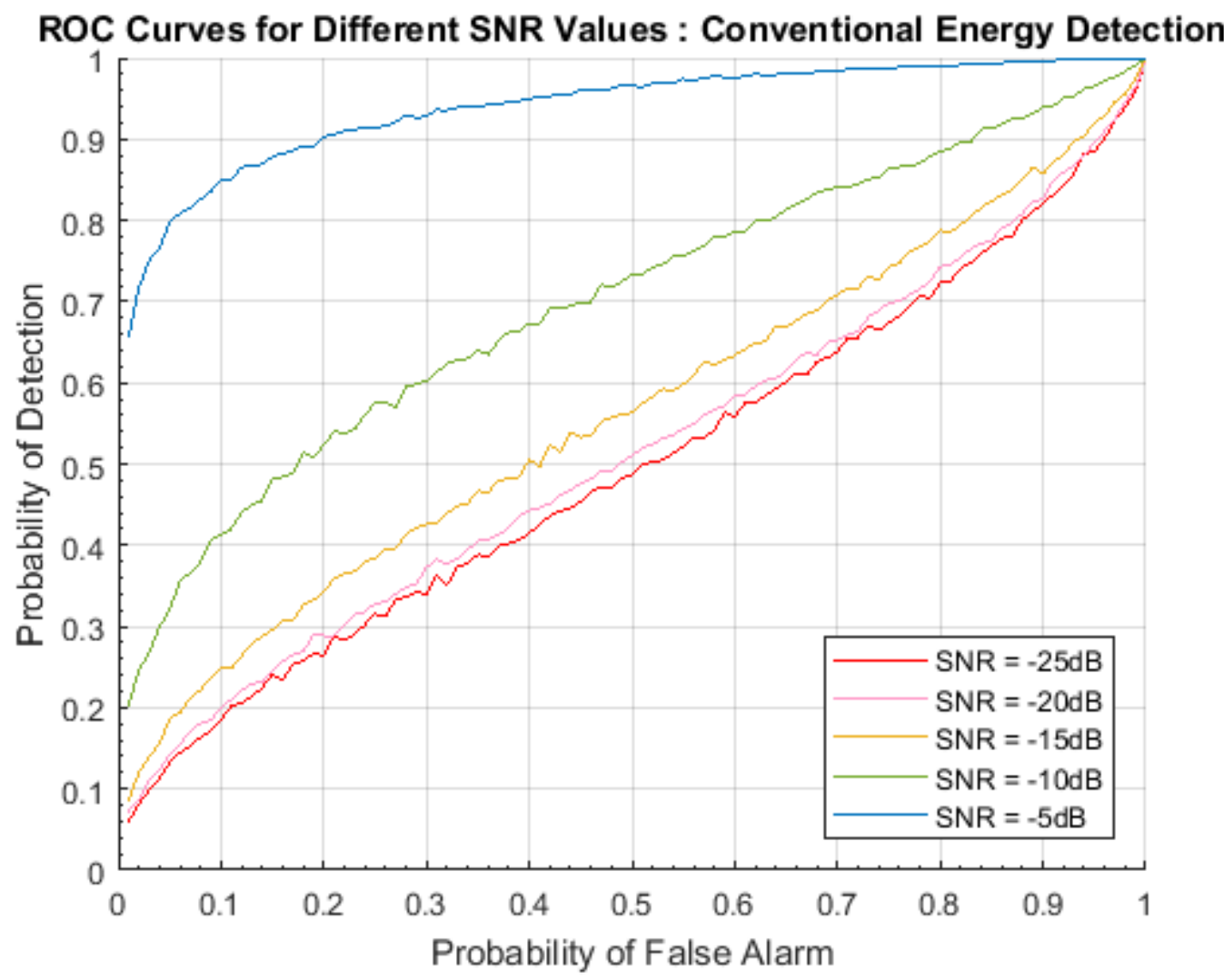

Figure 16: ROC Curves for Conventional Energy Detection at Different SNRs

The resulting graph formulated in Figure 16 is an ROC curve for a set of five different low signal-to-noise ratios (SNRs) within the range of $-25 \mathrm{~dB}$ to $-5 \mathrm{~dB}$. The value of $\mathrm{P}_{\mathrm{d}}$ varies from 0.18 to 0.84 for the conventional energy detection algorithm when $P_{f}=0.1$. For the ROC curve of the enhanced energy detection algorithm using matched filter, as shown in Figure 17, the value of $\mathrm{P}_{\mathrm{d}}$ varies from 0.925 to 0.985 when $\mathrm{P}_{\mathrm{f}}=0.1$. 


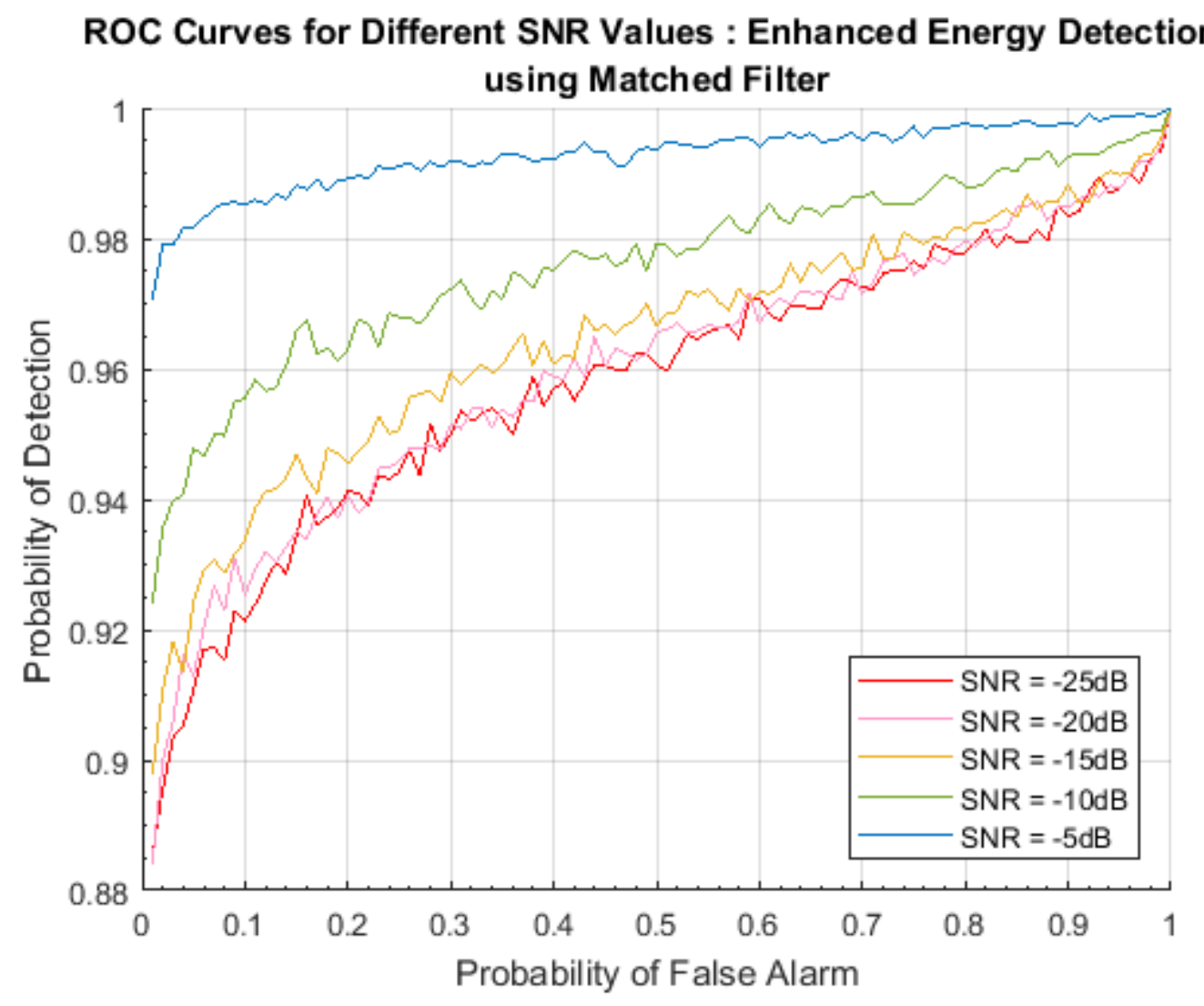

Figure 17: ROC Curves for Enhanced Energy Detection using Matched Filter at Different SNRs 


\section{CHAPTER 5}

\section{Conclusion and Future Work}

CR strives to accommodate the vastly increasing number of wireless applications [10], services and technologies of the 21st century through reliable spectrum sensing and dynamic allocation of spectrum bands, thus providing an enticing solution to the problem of spectral congestion and scarcity. Through statistical analysis and learning of the sensing accuracies, complexities, advantages and drawbacks, overall design and implementations, and the associated challenges, a thorough investigation was done by us in pursuance of determining an optimized method for spectrum sensing. This last chapter concludes our dissertation, and covers our plans and scope for future work.

\subsection{Conclusion}

The energy detector can be termed as a blind detector as it does not consider the nature of the signal, and the presence of a PU is ascertained only when the energy from the received signal levels crosses the energy threshold. It has low computational complexities and can detect the signal even if the user signal statistics are unfamiliar, however, it displays poor performance in low SNR environments and has the inability to distinguish between signal, noise, and interference. Our proposed novel algorithm can substantially improve the system performance of a conventional energy detector and help reduce unlicensed interference during spectrum sensing in cognitive radio networks. The simulation results show that our algorithm is justified in accordance to the IEEE 802.22 requirements, the results obtained from the enhanced energy detection algorithm aided by matched filter were quite satisfactory in terms of the performance metrics of $\mathrm{P}_{d}$ and $\mathrm{P}_{\mathrm{f}}$, since a high value of $\mathrm{P}_{\mathrm{d}}$ decreases the interference 
inflicted upon PUs and a low value of $\mathrm{P}_{\mathrm{f}}$ decreases the amount of missed spectral opportunities in the secondary network.

\subsection{Future Work}

Our plan is to take this dissertation further on the basis of making the algorithm more tuned and fit for real-world scenarios. We also hope to draw mightier comparisons of our devised algorithm with the state-of-the-arts, and hereby carry out a better performance analysis of the results obtained. Due to the lack of time and competence we have been unable to work for proper signal modulations, therefore our goal for the future is to apply Quadrature Amplitude Modulation (QAM) with Orthogonal Frequency-Division Multiplexing (OFDM) onto the signals for our proposed novel algorithm. By applying QAM with OFDM onto the signals, the bandwidth of each channel can be reduced by the number of multiplexed sub-carriers plus a guard band, so channel interference will be significantly less. Since there already exists a method and circuit for fine timing synchronization in the OFDM baseband receiver for IEEE 802.11A/G Wireless Local Area Network (WLAN) standard, and the protocol IEEE 802.22 is compatible with all other 802 protocols in the past, our algorithm will require minimal changes in the hardware and can be implemented. 


\section{REFERENCES}

[1] T. Y"ucek and H. Arslan, "A Survey of Spectrum Sensing Algorithms for Cognitive Radio Applications", IEEE communications surveys \& tutorials, vol. 11, no. 1, first quarter 2009.

[2] L. Ma, Y. Li, A. Demir, "Matched Filtering Assisted Energy Detection for Sensing Weak Primary User Signals," In: Proceeding of IEEE International Conference on Acoustics, Speech, and Signal Processing ICASSP 2012. Kyoto, Japan: 2012.

[3] E. Hossain, D. Niyato, Z. Han," Dynamic Spectrum Access and Management in Cognitive Radio Networks", 2009, [E-book], Available: www.cambridge.org.

[4] E. Peh and Y. C. Liang, "Optimization for Cooperative Sensing in Cognitive Radio Networks," IEEE WCNC, pp. 27-32, March 2007.

[5] Y.C. Liang, Y. Zeng, E.C.Y. Peh, and A.T. Hoang, "Sensing-Throughput Tradeoff for Cognitive Radio Networks", IEEE transactions on wireless communications, vol. 7, no. 4, April 2008.

[6] S. Atapattu, C. Tellambura and H. Jiang, "Energy Detection for Spectrum Sensing in Cognitive Radio", [E-Book], Available: http://www.springer.com/series/10028.

[7] Federal Communications Commission, "Facilitating Opportunities for Flexible, Efficient, and Reliable Spectrum Use Employing Cognitive Radio Technologies," December 30, 2003.

[8] J. Mitola, "An Integrated Agent Architecture for Software Defined Radio" in Doctor of technology Royal Inst. Technol. (KTH), Stockholm, Sweden, 2000.

[9] S. Haykin, "Cognitive Radio: Brain-Empowered Wireless Communications", IEEE journal on selected areas in communications, vol. 23, no. 2, February 2005. 
[10] M. Usman, K. Insoo, "Sensor Network-based Spectrum Sensing for Cognitive Radio Network", 2016 International Conference on Intelligent Systems Engineering (ICISE) 2016.

[11] S. Li and M. M. Kokar, "Flexible Adaptation in Cognitive Radios, Analog Circuits and Signal Processing”, [E-book], Available: http://www.springer.com/us/book/9781461409670.

[12] M. Song, C. Xin, Y. Zhao and X. Cheng, "Dynamic Spectrum Access: From Cognitive Radio to Network Radio", IEEE Wireless Communications, February 2012.

[13] Y. C. Liang, K. C. Chen, G. Y. Li and P. Mähönen, "Cognitive Radio Networking and Communications: An Overview", IEEE transactions on vehicular technology, vol. 60, no. 7, September 2011.

[14] J. Mitola and G. Q. Maguire, "Cognitive Radio: Making Software Radios More Personal," IEEE Personal Communications, vol. 6, no. 4, pp. 13-18, August 1999.

[15] M. Sherman, A. N. Mody, R. Martinez, and Christian Rodriguez," IEEE Standards Supporting Cognitive Radio and Networks, Dynamic Spectrum Access, and Coexistence", IEEE Communications Magazine, July 2008.

[16] A. Ghasemi, E. S. Sousa, "Spectrum Sensing in Cognitive Radio Networks: Requirements Challenges and Design Trade-offs", IEEE Communications Mag., vol. 46, pp. 32-39, April 2008.

[17] D. Cabric, S. Mishra, R. Brodersen, "Implementation Issues in Spectrum Sensing for Cognitive Radios", Proc. Asilomar Conf. on Signals Systems and Computers, vol. 1, pp. 772-776, November 2004. 
[18] S. Pandit and G. Singh, "Spectrum Sharing in Cognitive Radio NetworksMedium Access Control Protocol Based Approach", [E-book], Available: http://www.springer.com/gp/book/9783319531465.

[19] I. F. Akyildiz, B. F. Lo, R. Balakrishnan, "Cooperative Spectrum Sensing in Cognitive Radio Networks: A survey", Broadband Wireless Networking Laboratory, School of Electrical and Computer Engineering, Georgia Institute of Technology, Atlanta, GA, 30332, United States.

[20] H. Urkowitz, "Energy Detection of Unknown Deterministic Signals", Proceedings of the IEEE, vol. 55, no. 4, April 1967.

[21] D. H. Johnson (2006) Signal-to-noise ratio. Scholarpedia, 1(12):2088.

[22] M. Barkat, P. K. Vershney "On Adaptive Cell-Averaging CFAR Radar Signal Detection" (1987). Electrical Engineering and Computer Science - Dissertations. 276. Available: https://surface.syr.edu/eecs_etd/276.

[23] M. Rouaud, "Probability, Statistics and Estimation- Propagation of Uncertainties in Experimental Measurement”, July 2013, [E-book], Available: www.lulu.com.

[24] L. R. Welch, "Lower Bounds on the Maximum Cross Correlation of signals", IEEE Trans. Inform. Theory, vol. IT-20, pp. 397-399, May 1974.

[25] S. Shellhammer, G. Chouinard, "Spectrum Sensing Requirements Summary", Available: http://www.ieee802.org/22/Meeting_documents/2006_J-uly/22-06-008901-0000-Spectrum-Sensing-Requirements-Summary.doc, July 2006. 